\title{
Analyzing a Maximum Principle for Finite Horizon State Constrained Problems via Parametric Examples. Part 2: Problems with Bilateral State Constraints*
}

\author{
V.T. Huong` J.-C. Yao; and N.D. Yen ${ }^{\ddagger}$ \\ January 29, 2019 \\ (Dedicated to Professor Gue Myung Lee on the occasion of his 65th birthday)
}

\begin{abstract}
In the present paper, the maximum principle for finite horizon state constrained problems from the book by R. Vinter [Optimal Control, Birkhäuser, Boston, 2000; Theorem 9.3.1] is analyzed via parametric examples. The latter has origin in a recent paper by V. Basco, P. Cannarsa, and H. Frankowska, and resembles the optimal growth problem in mathematical economics. The solution existence of these parametric examples is established by invoking Filippov's existence theorem for Mayer problems. Since the maximum principle is only a necessary condition for local optimal processes, a large amount of additional investigations is needed to obtain a comprehensive synthesis of finitely many processes suspected for being local minimizers. Our analysis not only helps to understand the principle in depth, but also serves as a sample of applying it to meaningful prototypes of economic optimal growth models. Problems with unilateral state constraints have been studied in Part 1 of the paper. Problems with bilateral state constraints are addressed in this Part 2.
\end{abstract}

*In this research, Vu Thi Huong and Nguyen Dong Yen were supported by National Foundation for Science \& Technology Development (Vietnam) under grant number 101.01-2018.308.

$\dagger$ Institute of Mathematics, Vietnam Academy of Science and Technology, 18 Hoang Quoc Viet, Hanoi 10307, Vietnam; email: vthuong@math.ac.vn; huong263@gmail.com.

${ }^{\ddagger}$ Research Center for Interneural Computing, China Medical University Hospital, China Medical University, Taichung 40402, Taiwan; Email: yaojc@mail.cmu.edu.tw.

$\S$ Institute of Mathematics, Vietnam Academy of Science and Technology, 18 Hoang Quoc Viet, Hanoi 10307, Vietnam; email: ndyen@math.ac.vn. 
Keywords: Finite horizon optimal control problem, state constraint, maximum principle, solution existence theorem, function of bounded variation, Borel measurable function, Lebesgue-Stieltjes integral.

2010 Mathematics Subject Classification: 49K15, 49J15.

\section{Introduction}

It is well known that optimal control problems with state constraints are models of importance, but one usually faces with a lot of difficulties in analyzing them. These models have been considered since the early days of the optimal control theory. For instance, the whole Chapter VI of the classical work [1, pp. 257-316] is devoted to problems with restricted phase coordinates. There are various forms of the maximum principle for optimal control problems with state constraints; see, e.g., [2], where the relations between several forms are shown and a series of numerical illustrative examples have been solved.

To deal with state constraints, one has to use functions of bounded variation, Borel measurable functions, Lebesgue-Stieltjes integral, nonnegative measures on the $\sigma$-algebra of the Borel sets, the Riesz Representation Theorem for the space of continuous functions, and so on.

By using the maximum principle presented in [3, pp. 233-254], Phu [4, 5] has proposed an ingenious method called the method of region analysis to solve several classes of optimal control problems with one state and one control variable, which have both state and control constraints. Minimization problems of the Lagrange type were considered by the author and, among other things, it was assumed that integrand of the objective function is strictly convex with respect to the control variable. To be more precise, the author considered regular problems, i.e., the optimal control problems where the Pontryagin function is strictly convex with respect to the control variable.

In the present paper, the maximum principle for finite horizon state constrained problems from the book by Vinter [6, Theorem 9.3.1] is analyzed via parametric examples. The latter has origin in a recent paper by Basco, Cannarsa, and Frankowska [7, Example 1], and resembles the optimal growth problem in mathematical economics (see, e.g., [8, pp. 617-625]). The solution existence of these parametric examples, which are irregular optimal control problems in the sense of Phu [4, 5], is established by invoking Filippov's existence theorem for Mayer problems [9, Theorem 9.2.i and Section 9.4]. Since the maximum principle is only a necessary condition for local optimal processes, a large amount of additional investigations is needed to obtain a comprehensive synthesis of finitely many processes suspected for being local minimizers. Our analysis not only helps to understand the principle in depth, but also serves as a sample of applying it to meaningful prototypes of economic optimal growth 
models.

Note that the maximum principle for finite horizon state constrained problems in [6. Chapter 9] covers many known ones for smooth problems and allows us to deal with nonsmooth problems by using the Mordukhovich normal cone and the Mordukhovich subdifferential [10, 11, 12, which are also called the limiting normal cone and the limiting subdifferential. This principle is a necessary optimality condition which asserts the existence of a nontrivial multipliers set consisting of an absolutely continuous function, a function of bounded variation, a Borel measurable function, and a real number, such that the four conditions (i)-(iv) in Theorem 2.1 below are satisfied. The relationships between these conditions are worthy a detailed analysis. We will present such an analysis via three parametric examples of optimal control problems of the Langrange type, which have five parameters: the first one appears in the description of the objective function, the second one appears in the differential equation, the third one is the initial value, the fourth one is the initial time, and the fifth one is the terminal time. Observe that, in Example 1 of [7], the terminal time is infinity, the initial value and the initial time are fixed. Problems with unilateral state constraints have been studied in Part 1 (see [13]) of the paper. Problems with bilateral state constraints are addressed in this Part 2, which is organized as follows.

Section 2 presents some background materials including the above-mentioned maximum principle and Filippov's existence theorem for Mayer problems. Control problems with bilateral state constraints are studied in Section 3. Some concluding remarks are given in Section 4.

In comparison with Part 1, to deal with bilateral state constraints, herein we have to prove a series delicate lemmas and auxiliary propositions. Moreover, the synthesis of finitely many processes suspected for being local minimizers is rather sophisticated, and it requires a lot of refined arguments.

\section{Background Materials}

In this section, we give some notations, definitions, and results that will be used repeatedly in the sequel.

\subsection{Notations and Definitions}

The symbol $\mathbb{R}$ (resp., $\mathbb{N}$ ) denotes the set of real numbers (resp., the set of positive integers). The norm in the $n$-dimensional Euclidean space $\mathbb{R}^{n}$ is denoted by $\|$.$\| . For a subset C \subset \mathbb{R}^{n}$, we abbreviate its convex hull to co $C$. For a set-valued map $F: \mathbb{R}^{n} \rightrightarrows \mathbb{R}^{m}$, we call the set

$$
\operatorname{gph} F:=\left\{(x, y) \in \mathbb{R}^{n} \times \mathbb{R}^{m}: y \in F(x)\right\}
$$


the graph of $F$.

Let $\Omega \subset \mathbb{R}^{n}$ be a closed set and $\bar{v} \in \Omega$. The Fréchet (or regular) normal cone to $\Omega \subset \mathbb{R}^{n}$ at $\bar{v}$ is given by

$$
\widehat{N}_{\Omega}(\bar{v})=\left\{v^{\prime} \in \mathbb{R}^{n}: \limsup _{v \stackrel{\Omega}{\rightarrow}_{\bar{v}}} \frac{\left\langle v^{\prime}, v-\bar{v}\right\rangle}{\|v-\bar{v}\|} \leq 0\right\}
$$

where $v \stackrel{\Omega}{\rightarrow} \bar{v}$ means $v \rightarrow \bar{v}$ with $v \in \Omega$. The Mordukhovich (or limiting) normal cone to $\Omega$ at $\bar{v}$ is defined by

$$
N_{\Omega}(\bar{v})=\left\{v^{\prime} \in \mathbb{R}^{n}: \exists \text { sequences } v_{k} \rightarrow \bar{v}, v_{k}^{\prime} \rightarrow v^{\prime} \text { with } v_{k}^{\prime} \in \widehat{N}_{\Omega}\left(v_{k}\right) \text { for all } k \in \mathbb{N}\right\} .
$$

Given an extended real-valued function $\varphi: \mathbb{R}^{n} \rightarrow \mathbb{R} \cup\{-\infty,+\infty\}$, one defines the epigraph of $\varphi$ by epi $\varphi=\left\{(x, \mu) \in \mathbb{R}^{n} \times \mathbb{R}: \mu \geq \varphi(x)\right\}$. The Mordukhovich (or limiting) subdifferential of $\varphi$ at $\bar{x} \in \mathbb{R}^{n}$ with $|\varphi(\bar{x})|<\infty$ is defined by

$$
\partial \varphi(\bar{x})=\left\{x^{*} \in \mathbb{R}^{n}:\left(x^{*},-1\right) \in N((\bar{x}, \varphi(\bar{x})) ; \operatorname{epi} \varphi)\right\} .
$$

If $|\varphi(x)|=\infty$, then one puts $\partial \varphi(\bar{x})=\emptyset$. The reader is referred to [10, Chapter 1] and [12, Chapter 1] for comprehensive treatments of the Fréchet normal cone, the limiting normal cone, the limiting subdifferential, and the related calculus rules.

For a given segment $\left[t_{0}, T\right]$ of the real line, we denote the $\sigma$-algebra of its Lebesgue measurable subsets (resp., the $\sigma$-algebra of its Borel sets) by $\mathcal{L}$ (resp., $\mathcal{B}$ ). The Sobolev space $W^{1,1}\left(\left[t_{0}, T\right], \mathbb{R}^{n}\right)$ is the linear space of the absolutely continuous functions $x:\left[t_{0}, T\right] \rightarrow \mathbb{R}^{n}$ endowed with the norm

$$
\|x\|_{W^{1,1}}=\left\|x\left(t_{0}\right)\right\|+\int_{t_{0}}^{T}\|\dot{x}(t)\| d t
$$

(see, e.g., [14, p. 21] for this and another equivalent norm).

As in [6, p. 321], we consider the following finite horizon optimal control problem of the Mayer type, denoted by $\mathcal{M}$,

$$
\text { Minimize } g\left(x\left(t_{0}\right), x(T)\right) \text {, }
$$

over $x \in W^{1,1}\left(\left[t_{0}, T\right], \mathbb{R}^{n}\right)$ and measurable functions $u:\left[t_{0}, T\right] \rightarrow \mathbb{R}^{m}$ satisfying

$$
\begin{cases}\dot{x}(t)=f(t, x(t), u(t)), & \text { a.e. } t \in\left[t_{0}, T\right] \\ \left(x\left(t_{0}\right), x(T)\right) \in C & \text { a.e. } t \in\left[t_{0}, T\right] \\ u(t) \in U(t), & \forall t \in\left[t_{0}, T\right],\end{cases}
$$

where $\left[t_{0}, T\right]$ is a given interval, $g: \mathbb{R}^{n} \times \mathbb{R}^{n} \rightarrow \mathbb{R}, f:\left[t_{0}, T\right] \times \mathbb{R}^{n} \times \mathbb{R}^{m} \rightarrow \mathbb{R}^{n}$, and $h:\left[t_{0}, T\right] \times \mathbb{R}^{n} \rightarrow \mathbb{R}$ are given functions, $C \subset \mathbb{R}^{n} \times \mathbb{R}^{n}$ is a closed set, and $U:\left[t_{0}, T\right] \rightrightarrows \mathbb{R}^{m}$ is a set-valued map. 
A measurable function $u:\left[t_{0}, T\right] \rightarrow \mathbb{R}^{m}$ satisfying $u(t) \in U(t)$ a.e. $t \in\left[t_{0}, T\right]$ is called a control function. A process $(x, u)$ consists of a control function $u$ and an arc $x \in W^{1,1}\left(\left[t_{0}, T\right] ; \mathbb{R}^{n}\right)$ that is a solution to the differential equation in (2.2). A state trajectory $x$ is the first component of some process $(x, u)$. A process $(x, u)$ is called feasible if the state trajectory satisfies the endpoint constraint $\left(x\left(t_{0}\right), x(T)\right) \in C$ and the state constraint $h(t, x(t)) \leq 0$ for all $t \in\left[t_{0}, T\right]$.

Due to the appearance of the state constraint, the problem $\mathcal{M}$ in (2.1) $-(2.2)$ is said to be an optimal control problem with state constraints. But, if the inequality $h(t, x(t)) \leq 0$ is fulfilled for every $(t, x(t))$ with $t \in\left[t_{0}, T\right]$ and $x \in W^{1,1}\left(\left[t_{0}, T\right] ; \mathbb{R}^{n}\right.$ ) (for example, when $h$ is constant function having a fixed nonpositive value), i.e., the condition $h(t, x(t)) \leq 0$ for all $t \in\left[t_{0}, T\right]$ can be removed from (2.2), then one says that $\mathcal{M}$ an optimal control problem without state constraints.

The Hamiltonian $\mathcal{H}:\left[t_{0}, T\right] \times \mathbb{R}^{n} \times \mathbb{R}^{n} \times \mathbb{R}^{m} \rightarrow \mathbb{R}$ of $(2.2)$ is defined by

$$
\mathcal{H}(t, x, p, u):=p . f(t, x, u)=\sum_{i=1}^{n} p_{i} f_{i}(t, x, u) .
$$

Definition 2.1. A feasible process $(\bar{x}, \bar{u})$ is called a $W^{1,1}$ local minimizer for $\mathcal{M}$ if there exists $\delta>0$ such that $g\left(\bar{x}\left(t_{0}\right), \bar{x}(T)\right) \leq g\left(x\left(t_{0}\right), x(T)\right)$ for any feasible processes $(x, u)$ satisfying $\|\bar{x}-x\|_{W^{1,1}} \leq \delta$.

Definition 2.2. A feasible process $(\bar{x}, \bar{u})$ is called a $W^{1,1}$ global minimizer for $\mathcal{M}$ if, for any feasible processes $(x, u)$, one has $g\left(\bar{x}\left(t_{0}\right), \bar{x}(T)\right) \leq g\left(x\left(t_{0}\right), x(T)\right)$.

Definition 2.3 (See [6, p. 329]). The partial hybrid subdifferential $\partial_{x}^{>} h(t, x)$ of $h(t, x)$ w.r.t. $x$ is given by

$$
\begin{aligned}
\partial_{x}^{>} h(t, x):=\operatorname{co}\{\xi: & \text { there exists }\left(t_{i}, x_{i}\right) \stackrel{h}{\rightarrow}(t, x) \text { such that } \\
& \left.h\left(t_{k}, x_{k}\right)>0 \text { for all } k \text { and } \nabla_{x} h\left(t_{k}, x_{k}\right) \rightarrow \xi\right\},
\end{aligned}
$$

where $\left(t_{k}, x_{k}\right) \stackrel{h}{\rightarrow}(t, x)$ means that $\left(t_{k}, x_{k}\right) \rightarrow(t, x)$ and $h\left(t_{k}, x_{k}\right) \rightarrow h(t, x)$ as $k \rightarrow \infty$.

\subsection{A Maximum Principle for State Constrained Problems}

Due to the appearance of the state constraint $h(t, x(t)) \leq 0$ in $\mathcal{M}$, one has to introduce a multiplier that is an element in the topological dual $C^{*}\left(\left[t_{0}, T\right] ; \mathbb{R}\right)$ of the space of continuous functions $C\left(\left[t_{0}, T\right] ; \mathbb{R}\right)$ with the supremum norm. By the Riesz Representation Theorem (see, e.g., [14, Theorem 6, p. 374] and [15, Theorem 1, pp. 113-115]), any bounded linear functional $f$ on $C\left(\left[t_{0}, T\right] ; \mathbb{R}\right)$ can be uniquely represented in the form

$$
f(x)=\int_{\left[t_{0}, T\right]} x(t) d v(t),
$$


where $v$ is a function of bounded variation on $\left[t_{0}, T\right]$ which vanishes at $t_{0}$ and which are continuous from the right at every point $\tau \in\left(t_{0}, T\right)$, and $\int_{\left[t_{0}, T\right]} x(t) d v(t)$ is the RiemannStieltjes integral of $x$ with respect to $v$ (see, e.g., [14, p. 364]). The set of the elements of $C^{*}\left(\left[t_{0}, T\right] ; \mathbb{R}\right)$ which are given by nondecreasing functions $v$ is denoted by $C^{\oplus}\left(t_{0}, T\right)$.

Every $v \in C^{*}\left(\left[t_{0}, T\right] ; \mathbb{R}\right)$ corresponds to a finite regular measure, denoted by $\mu_{v}$, on the $\sigma$-algebra $\mathcal{B}$ of the Borel subsets of $\left[t_{0}, T\right]$ by the formula

$$
\mu_{v}(A):=\int_{\left[t_{0}, T\right]} \chi_{A}(t) d v(t),
$$

where $\chi_{A}(t)=1$ for $t \in A$ and $\chi_{A}(t)=0$ if $t \notin A$. Due to the correspondence $v \mapsto \mu_{v}$, we call every element $v \in C^{*}\left(\left[t_{0}, T\right] ; \mathbb{R}\right)$ a "measure" and identify $v$ with $\mu_{v}$. Clearly, the measure corresponding to each $v \in C^{\oplus}\left(t_{0}, T\right)$ is nonnegative.

The integrals $\int_{\left[t_{0}, t\right)} \nu(s) d \mu(s)$ and $\int_{\left[t_{0}, T\right]} \nu(s) d \mu(s)$ of a Borel measurable function $\nu$ in next theorem are understood in the sense of the Lebesgue-Stieltjes integration [14, p. 364].

The $\sigma$-algebra of the Borel sets in $\mathbb{R}^{m}$ is denoted by $\mathcal{B}^{m}$.

Theorem 2.1 (See [6, Theorem 9.3.1]). Let $(\bar{x}, \bar{u})$ be a $W^{1,1}$ local minimizer for $\mathcal{M}$. Assume that for some $\delta>0$, the following hypotheses are satisfied:

(H1) $f(., x,$.$) is \mathcal{L} \times \mathcal{B}^{m}$ measurable, for fixed $x$. There exists a Borel measurable function $k(.,):.\left[t_{0}, T\right] \times \mathbb{R}^{m} \rightarrow \mathbb{R}$ such that $t \mapsto k(t, \bar{u}(t))$ is integrable and

$$
\left\|f(t, x, u)-f\left(t, x^{\prime}, u\right)\right\| \leq k(t, u)\left\|x-x^{\prime}\right\|, \quad \forall x, x^{\prime} \in \bar{x}(t)+\delta \bar{B}, \forall u \in U(t), \text { a.e.; }
$$

(H2) gph $U$ is a Borel set in $\left[t_{0}, T\right] \times \mathbb{R}^{m}$;

(H3) $g$ is Lipschitz continuous on the ball $\left(\bar{x}\left(t_{0}\right), \bar{x}(T)\right)+\delta \bar{B}$;

(H4) $h$ is upper semicontinuous and there exists $K>0$ such that

$$
\left\|h(t, x)-h\left(t, x^{\prime}\right)\right\| \leq K\left\|x-x^{\prime}\right\|, \quad \forall x, x^{\prime} \in \bar{x}(t)+\delta \bar{B}, \forall t \in\left[t_{0}, T\right] .
$$

Then there exist $p \in W^{1,1}\left(\left[t_{0}, T\right] ; \mathbb{R}^{n}\right), \gamma \geq 0, \mu \in C^{\oplus}\left(t_{0}, T\right)$, and a Borel measurable function $\nu:\left[t_{0}, T\right] \rightarrow \mathbb{R}^{n}$ such that $(p, \mu, \gamma) \neq(0,0,0)$, and for $q(t):=p(t)+\eta(t)$ with $\eta(t):=\int_{\left[t_{0}, t\right)} \nu(s) d \mu(s)$ if $t \in\left[t_{0}, T\right)$ and $\eta(T):=\int_{\left[t_{0}, T\right]} \nu(s) d \mu(s)$, the following holds true:

(i) $\nu(t) \in \partial_{x}^{>} h(t, \bar{x}(t)) \mu-$ a.e.;

(ii) $-\dot{p}(t) \in \operatorname{co}_{x} \mathcal{H}(t, \bar{x}(t), q(t), \bar{u}(t))$ a.e.;

(iii) $\left(p\left(t_{0}\right),-q(T)\right) \in \gamma \partial g\left(\bar{x}\left(t_{0}\right), \bar{x}(T)\right)+N_{C}\left(\bar{x}\left(t_{0}\right), \bar{x}(T)\right)$;

(iv) $\mathcal{H}(t, \bar{x}(t), q(t), \bar{u}(t))=\max _{u \in U(t)} \mathcal{H}(t, \bar{x}(t), q(t), u)$ a.e. 


\subsection{Solution Existence in State Constrained Optimal Control}

To recall a solution existence theorem for optimal control problems with state constraints of the Mayer type, we will use the notations and concepts given in [9, Section 9.2]. Let $A$ be a subset of $\mathbb{R} \times \mathbb{R}^{n}$ and $U: A \rightrightarrows \mathbb{R}^{m}$ be a set-valued map defined on $A$. Let

$$
M:=\left\{(t, x, u) \in \mathbb{R} \times \mathbb{R}^{n} \times \mathbb{R}^{m}:(t, x) \in A, u \in U(t, x)\right\},
$$

and $f=\left(f_{1}, f_{2}, \ldots, f_{n}\right): M \rightarrow \mathbb{R}^{n}$ be a single-valued map defined on $M$. Let $B$ be a given subset of $\mathbb{R} \times \mathbb{R}^{n} \times \mathbb{R} \times \mathbb{R}^{n}$ and $g: B \rightarrow \mathbb{R}$ be a real function defined on $B$. Consider the optimal control problem of the Mayer type

$$
\text { Minimize } g\left(t_{0}, x\left(t_{0}\right), T, x(T)\right)
$$

over $x \in W^{1,1}\left(\left[t_{0}, T\right] ; \mathbb{R}^{n}\right)$ and measurable functions $u:\left[t_{0}, T\right] \rightarrow \mathbb{R}^{m}$ satisfying

$$
\begin{cases}\dot{x}(t)=f(t, x(t), u(t)), & \text { a.e. } t \in\left[t_{0}, T\right] \\ (t, x(t)) \in A, & \text { for all } t \in\left[t_{0}, T\right] \\ \left(t_{0}, x\left(t_{0}\right), T, x(T)\right) \in B & \\ u(t) \in U(t, x(t)), & \text { a.e. } t \in\left[t_{0}, T\right],\end{cases}
$$

where $\left[t_{0}, T\right]$ is a given interval. The problem (2.5)-(2.6) will be denoted by $\mathcal{M}_{1}$.

A feasible process for $\mathcal{M}_{1}$ is a pair of functions $(x, u)$ with $x:\left[t_{0}, T\right] \rightarrow \mathbb{R}^{n}$ being absolutely continuous on $\left[t_{0}, T\right], u:\left[t_{0}, T\right] \rightarrow \mathbb{R}^{m}$ being measurable, such that all the requirements in (2.6) are satisfied. If $(x, u)$ is a feasible process for $\mathcal{M}_{1}$, then $x$ is said to be a feasible trajectory, and $u$ a feasible control function for $\mathcal{M}_{1}$. The set of all feasible processes for $\mathcal{M}_{1}$ is denoted by $\Omega$.

Let $A_{0}=\left\{t \in \mathbb{R}: \exists x \in \mathbb{R}^{n}\right.$ s.t. $\left.(t, x) \in A\right\}$, i.e., $A_{0}$ is the projection of $A$ on the $t$-axis. Set

$$
A(t)=\left\{x \in \mathbb{R}^{n}:(t, x) \in A\right\} \quad\left(t \in A_{0}\right)
$$

and

$$
Q(t, x)=\left\{z \in \mathbb{R}^{n}: z=f(t, x, u), u \in U(t, x)\right\} \quad((t, x) \in A) .
$$

The forthcoming statement is called Filippov's Existence Theorem for Mayer problems.

Theorem 2.2 (see [9, Theorem 9.2.i and Section 9.4]). Suppose that $\Omega$ is nonempty, B is closed, $g$ is lower semicontinuous on $B, f$ is continuous on $M$ and, for almost every $t \in\left[t_{0}, T\right]$, the sets $Q(t, x), x \in A(t)$, are convex. Moreover, assume either that $A$ and $M$ are compact or that $A$ is not compact but closed and the following three conditions hold

(a) For any $\varepsilon \geq 0$, the set $M_{\varepsilon}:=\{(t, x, u) \in M:\|x\| \leq \varepsilon\}$ is compact; 
(b) There is a compact subset $P$ of $A$ such that every feasible trajectory $x$ of $\mathcal{M}_{1}$ passes through at least one point of $P$;

(c) There exists $c \geq 0$ such that

$$
x_{1} f_{1}(t, x, u)+x_{2} f_{2}(t, x, u)+\cdots+x_{n} f_{n}(t, x, u) \leq c\left(\|x\|^{2}+1\right) \quad \forall(t, x, u) \in M .
$$

Then, $\mathcal{M}_{1}$ has a $W^{1,1}$ global minimizer.

Clearly, condition (b) is satisfied if the initial point $\left(t_{0}, x\left(t_{0}\right)\right)$ or the end point $(T, x(T))$ is fixed. As shown in [9, p. 317], the following condition implies (c):

$\left(c_{0}\right)$ There exists $c \geq 0$ such that $\|f(t, x, u)\| \leq c(\|x\|+1)$ for all $(t, x, u) \in M$.

\section{Optimal Control Problems with Bilateral State Con- straints}

By $\left(F P_{3}\right)$ we denote the finite horizon optimal control problem of the Lagrange type

$$
\text { Minimize } J(x, u)=\int_{t_{0}}^{T}\left[-e^{-\lambda t}(x(t)+u(t))\right] d t
$$

over $x \in W^{1,1}\left(\left[t_{0}, T\right], \mathbb{R}\right)$ and measurable functions $u:\left[t_{0}, T\right] \rightarrow \mathbb{R}$ satisfying

$$
\begin{cases}\dot{x}(t)=-a u(t), & \text { a.e. } t \in\left[t_{0}, T\right] \\ x\left(t_{0}\right)=x_{0} & \\ u(t) \in[-1,1], & \text { a.e. } t \in\left[t_{0}, T\right] \\ -1 \leq x(t) \leq 1, & \forall t \in\left[t_{0}, T\right]\end{cases}
$$

with $a>\lambda>0, T>t_{0} \geq 0$, and $-1 \leq x_{0} \leq 1$ being given.

To treat $\left(F P_{3}\right)$ in (3.7)-(3.8) as a problem of the Mayer type, we set $x(t)=\left(x_{1}(t), x_{2}(t)\right)$, where $x_{1}(t)$ plays the role of $x(t)$ in $\left(F P_{3}\right)$ and

$$
x_{2}(t):=\int_{t_{0}}^{t}\left[-e^{-\lambda \tau}\left(x_{1}(\tau)+u(\tau)\right)\right] d \tau
$$

for all $t \in[0, T]$. Thus, $\left(F P_{3}\right)$ is equivalent to the problem

$$
\text { Minimize } x_{2}(T)
$$


over $x=\left(x_{1}, x_{2}\right) \in W^{1,1}\left(\left[t_{0}, T\right], \mathbb{R}^{2}\right)$ and measurable functions $u:\left[t_{0}, T\right] \rightarrow \mathbb{R}$ satisfying

$$
\begin{cases}\dot{x}_{1}(t)=-a u(t), & \text { a.e. } t \in\left[t_{0}, T\right] \\ \dot{x}_{2}(t)=-e^{-\lambda t}\left(x_{1}(t)+u(t)\right), & \text { a.e. } t \in\left[t_{0}, T\right] \\ \left(x\left(t_{0}\right), x(T)\right) \in\left\{\left(x_{0}, 0\right)\right\} \times \mathbb{R}^{2} & \\ u(t) \in[-1,1], & \text { a.e. } t \in\left[t_{0}, T\right] \\ -1 \leq x_{1}(t) \leq 1, & \forall t \in\left[t_{0}, T\right] .\end{cases}
$$

The problem (3.10)-(3.11) is abbreviated to $\left(F P_{3 a}\right)$.

\subsection{Solution Existence}

To verify that $\left(F P_{3 a}\right)$ is of the form $\mathcal{M}_{1}$ (see Subsection 2.3), one can choose $n=2, m=1$, $A=\left[t_{0}, T\right] \times[-1,1] \times \mathbb{R}, U(t, x)=[-1,1]$ for all $(t, x) \in A, B=\left\{t_{0}\right\} \times\left\{\left(x_{0}, 0\right)\right\} \times \mathbb{R} \times \mathbb{R}^{2}$, $g\left(t_{0}, x\left(t_{0}\right), T, x(T)\right)=x_{2}(T), M=A \times[-1,1], f(t, x, u)=\left(-a u,-e^{-\lambda t}\left(x_{1}+u\right)\right)$ for all $(t, x, u) \in M$. To show that $\left(F P_{3 a}\right)$ satisfies all the assumptions of Theorem 2.2 , we can use the arguments given in Subsection 3.1 in Part 1 ([13]), except those related to the convexity of the sets $Q(t, x)$ and the compactness of $M_{\varepsilon}$.

By the formula for $A$, one has $A_{0}=\left[t_{0}, T\right]$ and $A(t)=[-1,1] \times \mathbb{R}$ for all $t \in A_{0}$. Thus, the requirement in Theorem 2.2 on the convexity of the sets $Q(t, x), x \in A(t)$, for almost every $t \in\left[t_{0}, T\right]$ is satisfied. Since $M=\left[t_{0}, T\right] \times[-1,1] \times \mathbb{R} \times[-1,1]$, for any $\varepsilon \geq 0$, one has the expression $M_{\varepsilon}=\left\{(t, x, u) \in\left[t_{0}, T\right] \times[-1,1] \times \mathbb{R} \times[-1,1]:\|x\| \leq \varepsilon\right\}$, which justifies the compactness of $M_{\varepsilon}$.

Theorem 2.2 tells us that $\left(F P_{3 a}\right)$ has a $W^{1,1}$ global minimizer. Thus, by the equivalence of $\left(F P_{3}\right)$ and $\left(F P_{3 a}\right)$, we can assert that $\left(F P_{3}\right)$ has a $W^{1,1}$ global minimizer.

\subsection{Necessary Optimality Conditions}

To solve problem $\left(F P_{3}\right)$ by applying Theorem 2.1, note that $\left(F P_{3 a}\right)$ is in the form of $\mathcal{M}$ with $g(x, y)=y_{2}$,

$$
f(t, x, u)=\left(-a u,-e^{-\lambda t}\left(x_{1}+u\right)\right),
$$

$C=\left\{\left(x_{0}, 0\right)\right\} \times \mathbb{R}^{2}, U(t)=[-1,1]$, and $h(t, x)=\left|x_{1}\right|-1$ for all $t \in\left[t_{0}, T\right], x=\left(x_{1}, x_{2}\right) \in \mathbb{R}^{2}$,

$y=\left(y_{1}, y_{2}\right) \in \mathbb{R}^{2}$ and $u \in \mathbb{R}$. According to (2.3), the Hamiltonian of $\left(F P_{3 a}\right)$ is the function

$$
\mathcal{H}(t, x, p, u)=-\operatorname{aup}_{1}-e^{-\lambda t}\left(x_{1}+u\right) p_{2} \quad \forall(t, x, p, u) \in\left[t_{0}, T\right] \times \mathbb{R}^{2} \times \mathbb{R}^{2} \times \mathbb{R} .
$$


By (2.4), the partial hybrid subdifferential of $h$ at $(t, x) \in\left[t_{0}, T\right] \times \mathbb{R}^{2}$ is the set

$$
\partial_{x}^{>} h(t, x)= \begin{cases}\{(-1,0)\}, & \text { if } x_{1} \leq-1 \\ \emptyset, & \text { if }\left|x_{1}\right|<1 \\ \{(1,0)\}, & \text { if } x_{1} \geq 1\end{cases}
$$

Let $(\bar{x}, \bar{u})$ be a $W^{1,1}$ local minimizer for $\left(F P_{3 a}\right)$. Since the assumptions $(\mathrm{H} 1)-(\mathrm{H} 4)$ of Theorem 2.1 are satisfied for $\left(F P_{3 a}\right)$, by that theorem one can find $p \in W^{1,1}\left(\left[t_{0}, T\right] ; \mathbb{R}^{2}\right)$, $\gamma \geq 0, \mu \in C^{\oplus}\left(t_{0}, T\right)$, and a Borel measurable function $\nu:\left[t_{0}, T\right] \rightarrow \mathbb{R}^{2}$ such that $(p, \mu, \gamma) \neq$ $(0,0,0)$, and for $q(t):=p(t)+\eta(t)$ with

$$
\eta(t):=\int_{\left[t_{0}, t\right)} \nu(\tau) d \mu(\tau) \quad\left(\forall t \in\left[t_{0}, T\right)\right)
$$

and

$$
\eta(T):=\int_{\left[t_{0}, T\right]} \nu(\tau) d \mu(\tau),
$$

conditions (i)-(iv) in Theorem 2.1 hold true.

Condition (i): Note that

$$
\begin{aligned}
& \mu\left\{t \in\left[t_{0}, T\right]: \nu(t) \notin \partial_{x}^{>} h(t, \bar{x}(t))\right\} \\
& =\mu\left\{t \in\left[t_{0}, T\right]: \partial_{x}^{>} h(t, \bar{x}(t))=\emptyset\right\}+\mu\left\{t \in\left[t_{0}, T\right]: \partial_{x}^{>} h(t, \bar{x}(t)) \neq \emptyset, \nu(t) \notin \partial_{x}^{>} h(t, \bar{x}(t))\right\} .
\end{aligned}
$$

Since $-1 \leq x_{1}(t) \leq 1$ for every $t$, combining this with (3.13) gives

$$
\begin{aligned}
\mu\left\{t \in\left[t_{0}, T\right]: \nu(t) \notin \partial_{x}^{>} h(t, \bar{x}(t))\right\}= & \mu\left\{t \in\left[t_{0}, T\right]:-1<\bar{x}_{1}(t)<1\right\} \\
& +\mu\left\{t \in\left[t_{0}, T\right]: \bar{x}_{1}(t)=1, \nu(t) \neq(1,0)\right\} \\
& +\mu\left\{t \in\left[t_{0}, T\right]: \bar{x}_{1}(t)=-1, \nu(t) \neq(-1,0)\right\} .
\end{aligned}
$$

So, from (i) it follows that

$$
\begin{gathered}
\mu\left\{t \in\left[t_{0}, T\right]:-1<\bar{x}_{1}(t)<1\right\}=0, \\
\mu\left\{t \in\left[t_{0}, T\right]: \bar{x}_{1}(t)=1, \nu(t) \neq(1,0)\right\}=0, \\
\mu\left\{t \in\left[t_{0}, T\right]: \bar{x}_{1}(t)=-1, \nu(t) \neq(-1,0)\right\}=0 .
\end{gathered}
$$

Condition (ii): By (3.12), $\mathcal{H}$ is differentiable in $x$ and $\partial_{x} \mathcal{H}(t, x, p, u)=\left\{\left(-e^{-\lambda t} p_{2}, 0\right)\right\}$ for all $(t, x, p, u) \in\left[t_{0}, T\right] \times \mathbb{R}^{2} \times \mathbb{R}^{2} \times \mathbb{R}$. Thus, (ii) implies that $-\dot{p}(t)=\left(-e^{-\lambda t} q_{2}(t), 0\right)$ for a.e. $t \in\left[t_{0}, T\right]$. Hence, $\dot{p}_{1}(t)=e^{-\lambda t} q_{2}(t)$ for a.e. $t \in\left[t_{0}, T\right]$ and $p_{2}(t)$ is a constant for all $t \in\left[t_{0}, T\right]$. 
Condition (iii): By the formulas for $g$ and $C, \partial g\left(\bar{x}\left(t_{0}\right), \bar{x}(T)\right)=\{(0,0,0,1)\}$ and $N_{C}\left(\bar{x}\left(t_{0}\right), \bar{x}(T)\right)=\mathbb{R}^{2} \times\{(0,0)\}$. Thus, (iii) yields

$$
\left(p\left(t_{0}\right),-q(T)\right) \in\{(0,0,0, \gamma)\}+\mathbb{R}^{2} \times\{(0,0)\},
$$

which means that $q_{1}(T)=0$ and $q_{2}(T)=-\gamma$.

Condition (iv): By (3.12), from (iv) one gets

$-a \bar{u}(t) q_{1}(t)-e^{-\lambda t}\left[\bar{x}_{1}(t)+\bar{u}(t)\right] q_{2}(t)=\max _{u \in[-1,1]}\left\{-a u q_{1}(t)-e^{-\lambda t}\left[\bar{x}_{1}(t)+u\right] q_{2}(t)\right\}$ a.e. $t \in\left[t_{0}, T\right]$

or, equivalently,

$$
\left[a q_{1}(t)+e^{-\lambda t} q_{2}(t)\right] \bar{u}(t)=\min _{u \in[-1,1]}\left\{\left[a q_{1}(t)+e^{-\lambda t} q_{2}(t)\right] u\right\} \text { a.e. } t \in\left[t_{0}, T\right] .
$$

If the curve $\bar{x}_{1}(t)$ remains in the interior of the domain $[-1,1]$ for all $t$ from an open interval $\left(\tau_{1}, \tau_{2}\right)$ of the time axis and touches the boundary of the domain at the moments $\tau_{1}$ and $\tau_{2}$, then it must have some special form. A formal formulation of this observation is as follows.

Proposition 3.1. Suppose that $\left[\tau_{1}, \tau_{2}\right], \tau_{1}<\tau_{2}$, is a subsegment of $\left[t_{0}, T\right]$ with $\bar{x}_{1}(t) \in(-1,1)$ for all $t \in\left(\tau_{1}, \tau_{2}\right)$. Then, next statements hold true.

S1) If $\bar{x}_{1}\left(\tau_{1}\right)=-1$ and $\bar{x}_{1}\left(\tau_{2}\right)=1$, then $\tau_{2}-\tau_{1}=2 a^{-1}$ and

$$
\bar{x}_{1}(t)=-1+a\left(t-\tau_{1}\right), \quad t \in\left[\tau_{1}, \tau_{2}\right] .
$$

S2) If $\bar{x}_{1}\left(\tau_{1}\right)=1$ and $\bar{x}_{1}\left(\tau_{2}\right)=-1$, then $\tau_{2}-\tau_{1}=2 a^{-1}$ and

$$
\bar{x}_{1}(t)=1-a\left(t-\tau_{1}\right), \quad t \in\left[\tau_{1}, \tau_{2}\right] .
$$

S3) If $\bar{x}_{1}\left(\tau_{1}\right)=\bar{x}_{1}\left(\tau_{2}\right)=-1$, then $\tau_{2}-\tau_{1}<4 a^{-1}$ and

$$
\bar{x}_{1}(t)= \begin{cases}-1+a\left(t-\tau_{1}\right), & t \in\left[\tau_{1}, \hat{t}\right] \\ -1-a\left(t-\tau_{2}\right), & t \in\left(\hat{t}, \tau_{2}\right],\end{cases}
$$

where $\hat{t}:=\left(\tau_{1}+\tau_{2}\right) / 2$.

S4) The situation where $\bar{x}_{1}\left(\tau_{1}\right)=\bar{x}_{1}\left(\tau_{2}\right)=1$ cannot happen.

Proof. Choose $\varepsilon_{1}>$ and $\varepsilon_{2}>0$ small enough so as $\left[\tau_{1}+\varepsilon_{1}, \tau_{2}-\varepsilon_{2}\right] \subset\left[\tau_{1}, \tau_{2}\right]$. Then, $\bar{x}_{1}(t) \in(-1,1)$ for all $t \in\left[\tau_{1}+\varepsilon_{1}, \tau_{2}-\varepsilon_{2}\right]$, i.e., $h(t, \bar{x}(t))<0$ for all $t \in\left[\tau_{1}+\varepsilon_{1}, \tau_{2}-\varepsilon_{2}\right]$. Thus, applying Proposition 4.3 in Part 1 ([13]) with $\left(F P_{3 a}\right)$ in the place of $\left(F P_{2 a}\right)$ in its 
formulation, one finds that the formula for $\bar{x}_{1}($.$) on \left[\tau_{1}+\varepsilon_{1}, \tau_{2}-\varepsilon_{2}\right]$ belongs to one of the following categories $\mathrm{C} 1-\mathrm{C} 3$ :

$$
\begin{array}{ll}
\bar{x}_{1}(t)=\bar{x}_{1}\left(\tau_{1}+\varepsilon_{1}\right)+a\left(t-\tau_{1}-\varepsilon_{1}\right), & t \in\left[\tau_{1}+\varepsilon_{1}, \tau_{2}-\varepsilon_{2}\right], \\
\bar{x}_{1}(t)=\bar{x}_{1}\left(\tau_{1}+\varepsilon_{1}\right)-a\left(t-\tau_{1}-\varepsilon_{1}\right), & t \in\left[\tau_{1}+\varepsilon_{1}, \tau_{2}-\varepsilon_{2}\right],
\end{array}
$$

and

$$
\bar{x}_{1}(t)= \begin{cases}\bar{x}_{1}\left(\tau_{1}+\varepsilon_{1}\right)+a\left(t-\tau_{1}-\varepsilon_{1}\right), & t \in\left[\tau_{1}+\varepsilon_{1}, t_{\zeta}\right] \\ \bar{x}_{1}\left(t_{\zeta}\right)-a\left(t-t_{\zeta}\right), & t \in\left(t_{\zeta}, \tau_{2}-\varepsilon_{2}\right],\end{cases}
$$

where $t_{\zeta}$ is some point in $\left(\tau_{1}+\varepsilon_{1}, \tau_{2}-\varepsilon_{2}\right)$.

To prove the statement $\mathrm{S} 1$, let $\varepsilon_{2}=k^{-1}$ with $k$ being a positive integer, as large as $k^{-1} \in\left(\tau_{1}+\varepsilon_{1}, \tau_{2}\right)$. Since for each $k$ the formula for $\bar{x}_{1}($.$) on \left[\tau_{1}+\varepsilon_{1}, \tau_{2}-k^{-1}\right]$ must be of the three types $\mathrm{C} 1-\mathrm{C} 3$, by the Dirichlet principle there must exist a subsequence $\left\{k^{\prime}\right\}$ of $\{k\}$ such that the corresponding formulas belong to a fixed category. If the latter is happens to be $\mathrm{C} 2$, then by the continuity of $\bar{x}_{1}($.$) one has$

$\bar{x}_{1}\left(\tau_{2}\right)=\lim _{k^{\prime} \rightarrow \infty} \bar{x}_{1}\left(\tau_{2}-\frac{1}{k^{\prime}}\right)=\lim _{k^{\prime} \rightarrow \infty}\left[\bar{x}_{1}\left(\tau_{1}+\varepsilon_{1}\right)-a\left(\tau_{2}-\frac{1}{k^{\prime}}-\tau_{1}-\varepsilon_{1}\right)\right]=\bar{x}_{1}\left(\tau_{1}+\varepsilon_{1}\right)-a\left(\tau_{2}-\tau_{1}-\varepsilon_{1}\right)$.

This is impossible, because $\bar{x}_{1}\left(\tau_{2}\right)=1$. Similarly, the situation where the fixed category is C3 must also be excluded. In the case where the formulas for $\bar{x}_{1}($.$) belong to the category \mathrm{C} 1$, we have

$$
\bar{x}_{1}(t)=\bar{x}_{1}\left(\tau_{1}+\varepsilon_{1}\right)+a\left(t-\tau_{1}-\varepsilon_{1}\right), \quad t \in\left[\tau_{1}+\varepsilon_{1}, \tau_{2}\right] .
$$

Now, letting $\varepsilon_{1}$ tend to zero and using continuity of $\bar{x}_{1}($.$) , we obtain$

$$
\bar{x}_{1}(t)=\bar{x}_{1}\left(\tau_{1}\right)+a\left(t-\tau_{1}\right), \quad t \in\left[\tau_{1}, \tau_{2}\right] .
$$

As $\bar{x}_{1}\left(\tau_{1}\right)=-1$, the statement $\mathrm{S} 1$ is proved.

The statements S2 and S3 are proved similarly.

To prove the assertion S4, it suffices to apply the arguments of the second part of the analysis of Subcase 4b in Subsection 4.2 in Part 1 ([13]).

The forthcoming technical lemma will be in use very frequently.

Lemma 3.2. Given any $t_{1}, t_{2} \in\left[t_{0}, T\right], t_{1}<t_{2}$, one puts

$$
\left.J(x, u)\right|_{\left[t_{1}, t_{2}\right]}:=\int_{t_{1}}^{t_{2}}\left[-e^{-\lambda t}\left(x_{1}(t)+u(t)\right)\right] d t
$$

for any feasible process $(x, u)$ of $\left(F P_{3 a}\right)$. If $(\widetilde{x}, \widetilde{u})$ and $(\check{x}, \check{u})$ are feasible processes for $\left(F P_{3 a}\right)$ with $\widetilde{x}_{1}(t)=1$ for all $t \in\left[t_{1}, t_{2}\right]$ and

$$
\check{x}_{1}(t)= \begin{cases}1-a\left(t-t_{1}\right), & t \in\left[t_{1}, \check{t}\right] \\ 1+a\left(t-t_{2}\right), & t \in\left(\check{t}, t_{2}\right]\end{cases}
$$


where $\check{t}:=2^{-1}\left(t_{1}+t_{2}\right)$, then one has

$$
\left.J(\check{x}, \check{u})\right|_{\left[t_{1}, t_{2}\right]}-\left.J(\widetilde{x}, \widetilde{u})\right|_{\left[t_{1}, t_{2}\right]}=\frac{1}{\lambda}\left(\frac{a}{\lambda}-1\right) \Delta\left(t_{1}, t_{2}\right)
$$

with

$$
\Delta\left(t_{1}, t_{2}\right):=e^{-\lambda t_{1}}-2 e^{-\frac{1}{2} \lambda\left(t_{1}+t_{2}\right)}+e^{-\lambda t_{2}} .
$$

Besides, it holds that $\Delta\left(t_{1}, t_{2}\right)>0$ and $\left.J(\check{x}, \check{u})\right|_{\left[t_{1}, t_{2}\right]}>\left.J(\widetilde{x}, \widetilde{u})\right|_{\left[t_{1}, t_{2}\right]}$.

Proof. Using the equation $\dot{x}_{1}(t)=-a u(t)$ in (3.11), which is fulfilled for almost all $t \in\left[t_{0}, T\right]$, and the assumed properties of the processes $(\widetilde{x}, \widetilde{u})$ and $(\check{x}, \check{u})$, we have $\widetilde{u}(t)=0$ for almost all $t \in\left[t_{1}, t_{2}\right]$ and

$$
\check{u}(t)= \begin{cases}1, & \text { a.e. } t \in\left[t_{1}, \check{t}\right] \\ -1, & \text { a.e. } t \in\left(\check{t}, t_{2}\right] .\end{cases}
$$

Since $\check{x}(\cdot)$ is a feasible trajectory for $\left(F P_{3 a}\right)$, one has $\check{x}(\check{t}) \geq-1$, i.e., $t_{2}-t_{1} \leq 4 a^{-1}$.

By the formulas for $\widetilde{x}_{1}$ and $\widetilde{u}$ on $\left[t_{1}, t_{2}\right]$,

$$
\left.J(\widetilde{x}, \widetilde{u})\right|_{\left[t_{1}, t_{2}\right]}=\int_{t_{1}}^{t_{2}}\left[-e^{-\lambda t}\left(\widetilde{x}_{1}(t)+\widetilde{u}(t)\right)\right] d t=-\int_{t_{1}}^{t_{2}} e^{-\lambda t} d t=\frac{1}{\lambda} e^{-\lambda t_{2}}-\frac{1}{\lambda} e^{-\lambda t_{1}} .
$$

Similarly, from the formulas for $\check{x}_{1}$ and $\check{u}$ on $\left[t_{1}, t_{2}\right]$ it follows that

$$
\begin{aligned}
\left.J(\check{x}, \check{u})\right|_{\left[t_{1}, t_{2}\right]}= & \int_{t_{1}}^{t_{2}}\left[-e^{-\lambda t}\left(\check{x}_{1}(t)+\check{u}(t)\right)\right] d t \\
= & \int_{t_{1}}^{\check{t}}\left[-e^{-\lambda t}\left(\left(1-a\left(t-t_{1}\right)\right)+1\right)\right] d t \\
& +\int_{\check{t}}^{t_{2}}\left[-e^{-\lambda t}\left(\left(1+a\left(t-t_{2}\right)\right)-1\right)\right] d t \\
= & \int_{t_{1}}^{\check{t}} e^{-\lambda t}\left[a\left(t-t_{1}\right)-2\right] d t-\int_{\check{t}}^{t_{2}} e^{-\lambda t} a\left(t-t_{2}\right) d t .
\end{aligned}
$$

Denote the last two integrals respectively by $I_{1}$ and $I_{2}$. Then, $\left.J(\check{x}, \check{u})\right|_{\left[t_{1}, t_{2}\right]}=I_{1}-I_{2}$. By regrouping and applying the formula for integration by parts, one has

$$
\begin{aligned}
I_{1} & =-\frac{a}{\lambda} \int_{t_{1}}^{\check{t}}\left(t-t_{1}\right) d\left(e^{-\lambda t}\right)-2 \int_{t_{1}}^{\check{t}} e^{-\lambda t} d t \\
& \left.=-\left.\frac{a}{\lambda}\left[\left(t-t_{1}\right) e^{-\lambda t}\right]\right|_{t_{1}} ^{\check{t}}-\int_{t_{1}}^{\check{t}} e^{-\lambda t} d t\right]-2 \int_{t_{1}}^{\check{t}} e^{-\lambda t} d t \\
& =\left(\frac{a}{\lambda}-2\right) \int_{t_{1}}^{\check{t}} e^{-\lambda t} d t-\frac{a}{2 \lambda}\left(t_{2}-t_{1}\right) e^{-\lambda \check{t}} \\
& =\left(\frac{2}{\lambda}-\frac{a}{\lambda^{2}}\right)\left(e^{-\lambda \check{t}}-e^{-\lambda t_{1}}\right)-\frac{a}{2 \lambda}\left(t_{2}-t_{1}\right) e^{-\lambda \check{t}} .
\end{aligned}
$$


Similarly,

$$
\begin{aligned}
I_{2} & =-\frac{a}{\lambda} \int_{\check{t}}^{t_{2}}\left(t-t_{2}\right) d\left(e^{-\lambda t}\right) \\
& =\left.\frac{a}{\lambda}\left[\left(t-t_{2}\right) e^{-\lambda t}\right]\right|_{t_{2}} ^{\check{t}}+\frac{a}{\lambda}\left[\int_{\check{t}}^{t_{2}} e^{-\lambda t} d t\right] \\
& =-\frac{a}{2 \lambda}\left(t_{2}-t_{1}\right) e^{-\lambda \check{t}}-\frac{a}{\lambda^{2}}\left[e^{-\lambda t_{2}}-e^{-\lambda \check{t}}\right] .
\end{aligned}
$$

Thus,

$$
\begin{aligned}
\left.J(\check{x}, \check{u})\right|_{\left[t_{1}, t_{2}\right]}= & \left(\frac{2}{\lambda}-\frac{a}{\lambda^{2}}\right)\left(e^{-\lambda \check{t}}-e^{-\lambda t_{1}}\right)-\frac{a}{2 \lambda}\left(t_{2}-t_{1}\right) e^{-\lambda \check{t}}+\frac{a}{2 \lambda}\left(t_{2}-t_{1}\right) e^{-\lambda \check{t}} \\
& +\frac{a}{\lambda^{2}}\left[e^{-\lambda t_{2}}-e^{-\lambda \check{t}]}\right. \\
= & \left(\frac{2}{\lambda}-\frac{2 a}{\lambda^{2}}\right) e^{-\lambda \check{t}}+\left(\frac{a}{\lambda^{2}}-\frac{2}{\lambda}\right) e^{-\lambda t_{1}}+\frac{a}{\lambda^{2}} e^{-\lambda t_{2}} .
\end{aligned}
$$

Therefore,

$$
\begin{aligned}
\left.J(\check{x}, \check{u})\right|_{\left[t_{1}, t_{2}\right]}-\left.J(\widetilde{x}, \widetilde{u})\right|_{\left[t_{1}, t_{2}\right]} & =\left(\frac{2}{\lambda}-\frac{2 a}{\lambda^{2}}\right) e^{-\lambda \check{t}}+\left(\frac{a}{\lambda^{2}}-\frac{1}{\lambda}\right) e^{-\lambda t_{1}}+\left(\frac{a}{\lambda^{2}}-\frac{1}{\lambda}\right) e^{-\lambda t_{2}} \\
& =\frac{1}{\lambda}\left(\frac{a}{\lambda}-1\right)\left(e^{-\lambda t_{1}}-2 e^{-\lambda \check{t}}+e^{-\lambda t_{2}}\right) .
\end{aligned}
$$

Thus, formula (3.19) is proved. To obtain the second assertion of the lemma, put $\psi(t)=e^{-\lambda t}$ for all $t \in \mathbb{R}$. Since $\psi^{\prime \prime}(t)>0$ for every $t$, the function $\psi$ is strictly convex. So,

$$
\psi\left(\frac{1}{2} t_{1}+\frac{1}{2} t_{2}\right)<\frac{1}{2} \psi\left(t_{1}\right)+\frac{1}{2} \psi\left(t_{2}\right) .
$$

It follows that $\Delta\left(t_{1}, t_{2}\right)>0$ for any $t_{1}<t_{2}$. Combining this with (3.21) and the inequality $\frac{a}{\lambda}-1>0$, we obtain the strict inequality $\left.J(\check{x}, \check{u})\right|_{\left[t_{1}, t_{2}\right]}>\left.J(\widetilde{x}, \widetilde{u})\right|_{\left[t_{1}, t_{2}\right]}$.

The following analogue of Lemma 3.2 will be used latter on.

Lemma 3.3. Let $t_{1}, t_{2}$ be as in Lemma 3.2. Let $\left.J(x, u)\right|_{\left[t_{1}, t_{2}\right]}$ and $\Delta\left(t_{1}, t_{2}\right)$ be defined, respectively, by (3.17) and (3.20). If $(\widetilde{x}, \widetilde{u})$ and $(\hat{x}, \hat{u})$ are feasible processes for $\left(F P_{3 a}\right)$ with $\widetilde{x}_{1}(t)=-1$ for all $t \in\left[t_{1}, t_{2}\right]$ and

$$
\hat{x}_{1}(t)= \begin{cases}-1+a\left(t-t_{1}\right), & t \in\left[t_{1}, \hat{t}\right] \\ -1-a\left(t-t_{2}\right), & t \in\left(\hat{t}, t_{2}\right],\end{cases}
$$

where $\hat{t}:=2^{-1}\left(t_{1}+t_{2}\right)$, then one has

$$
\left.J(\hat{x}, \hat{u})\right|_{\left[t_{1}, t_{2}\right]}-\left.J(\widetilde{x}, \widetilde{u})\right|_{\left[t_{1}, t_{2}\right]}=-\frac{1}{\lambda}\left(\frac{a}{\lambda}-1\right) \Delta\left(t_{1}, t_{2}\right) .
$$

Therefore, $\left.J(\hat{x}, \hat{u})\right|_{\left[t_{1}, t_{2}\right]}<\left.J(\widetilde{x}, \widetilde{u})\right|_{\left[t_{1}, t_{2}\right]}$. 
Proof. By (3.11), from our assumptions it follows that $\widetilde{u}(t)=0$ for almost all $t \in\left[t_{1}, t_{2}\right]$ and

$$
\hat{u}(t)= \begin{cases}-1, & \text { a.e. } t \in\left[t_{1}, \hat{t}\right] \\ 1, & \text { a.e. } t \in\left(\hat{t}, t_{2}\right]\end{cases}
$$

Since $\hat{x}(\cdot)$ is a feasible trajectory for $\left(F P_{3 a}\right)$, one has $\hat{x}(\hat{t}) \leq 1$, i.e., $t_{2}-t_{1} \leq 4 a^{-1}$. One has

$$
\left.J(\widetilde{x}, \widetilde{u})\right|_{\left[t_{1}, t_{2}\right]}=\int_{t_{1}}^{t_{2}}\left[-e^{-\lambda t}\left(\widetilde{x}_{1}(t)+\widetilde{u}(t)\right)\right] d t=\int_{t_{1}}^{t_{2}} e^{-\lambda t} d t=-\frac{1}{\lambda} e^{-\lambda t_{2}}+\frac{1}{\lambda} e^{-\lambda t_{1}} .
$$

Besides, the formulas for $\hat{x}_{1}$ and $\hat{u}$ on $\left[t_{1}, t_{2}\right]$ imply that

$$
\begin{aligned}
\left.J(\hat{x}, \hat{u})\right|_{\left[t_{1}, t_{2}\right]}= & \int_{t_{1}}^{\hat{t}}\left[-e^{-\lambda t}\left(\left(-1+a\left(t-t_{1}\right)\right)-1\right)\right] d t \\
& +\int_{\hat{t}}^{t_{2}}\left[-e^{-\lambda t}\left(\left(-1-a\left(t-t_{2}\right)\right)+1\right)\right] d t \\
= & -\int_{t_{1}}^{t \hat{t}} e^{-\lambda t}\left[a\left(t-t_{1}\right)-2\right] d t+\int_{\hat{t}}^{t_{2}} e^{-\lambda t} a\left(t-t_{2}\right) d t .
\end{aligned}
$$

Thus, changing the sign of the expression $\left.J(\hat{x}, \hat{u})\right|_{\left[t_{1}, t_{2}\right]}-\left.J(\widetilde{x}, \widetilde{u})\right|_{\left[t_{1}, t_{2}\right]}$ we get the expression on the left-hand-side of (3.19). So, the desired results follow from Lemma 3.2

We will need two more lemmas.

Lemma 3.4. Consider the function $\Delta: \mathbb{R}^{2} \rightarrow \mathbb{R}$ defined by (3.20). For any $t_{1}, t_{2} \in \mathbb{R}$ with $t_{1}<t_{2}$ and for any $\bar{\varepsilon} \in\left(0, t_{2}-t_{1}\right)$, one has

$$
\Delta\left(t_{1}+\bar{\varepsilon}, t_{2}\right)<\Delta\left(t_{1}, t_{2}\right)
$$

and

$$
\Delta\left(t_{1}, t_{2}\right)>\Delta\left(t_{1}, t_{1}+\bar{\varepsilon}\right)+\Delta\left(t_{1}+\bar{\varepsilon}, t_{2}\right)
$$

Proof. Fix a value $\bar{\varepsilon} \in\left(0, t_{2}-t_{1}\right)$. To obtain (3.22), consider the function $\psi_{1}(\varepsilon):=\Delta\left(t_{1}+\varepsilon, t_{2}\right)$ of the variable $\varepsilon \in \mathbb{R}$. Since $\psi_{1}(\varepsilon)=e^{-\lambda\left(t_{1}+\varepsilon\right)}-2 e^{-\frac{1}{2} \lambda\left(t_{1}+\varepsilon+t_{2}\right)}+e^{-\lambda t_{2}}$, one sees that $\psi_{1}($.) is continuously differentiable on $\mathbb{R}$ and $\psi_{1}^{\prime}(\varepsilon)=\lambda\left(e^{-\frac{1}{2} \lambda\left(t_{1}+\varepsilon+t_{2}\right)}-e^{-\lambda\left(t_{1}+\varepsilon\right)}\right)$. As the function $r(t):=e^{-\lambda t}$ is strictly decreasing on $\mathbb{R}$, the last equality implies that $\psi_{1}^{\prime}(\varepsilon)<0$ for every $\varepsilon \in\left[0, t_{2}-t_{1}\right)$. Hence, the function $\psi_{1}($.$) is strictly decreasing on \left[0, t_{2}-t_{1}\right)$. So, the inequality 3.22 is valid.

To obtain (3.23), observe from (3.20) that

$$
\begin{aligned}
& \Delta\left(t_{1}, t_{2}\right)-\Delta\left(t_{1}, t_{1}+\bar{\varepsilon}\right)-\Delta\left(t_{1}+\bar{\varepsilon}, t_{2}\right) \\
& =e^{-\lambda t_{1}}-2 e^{-\lambda \frac{t_{1}+t_{2}}{2}}+e^{-\lambda t_{2}}-\left(e^{-\lambda t_{1}}-2 e^{-\lambda\left(t_{1}+\frac{\bar{\varepsilon}}{2}\right)}+e^{-\lambda\left(t_{1}+\bar{\varepsilon}\right)}\right) \\
& \quad-\left(e^{-\lambda\left(t_{1}+\bar{\varepsilon}\right)}-2 e^{-\lambda\left(\frac{t_{1}+t_{2}}{2}+\frac{\bar{\varepsilon}}{2}\right)}+e^{-\lambda t_{2}}\right) \\
& =2\left[e^{-\lambda\left(\frac{t_{1}+t_{2}}{2}+\frac{\bar{\varepsilon}}{2}\right)}-e^{-\lambda \frac{t_{1}+t_{2}}{2}}\right]-2\left[e^{-\lambda\left(t_{1}+\bar{\varepsilon}\right)}-e^{-\lambda\left(t_{1}+\frac{\bar{\varepsilon}}{2}\right)}\right]
\end{aligned}
$$


Applying the classical mean value theorem to the differentiable function $r(t)=e^{-\lambda t}$, one can find $\tau_{1} \in\left(t_{1}+\frac{\bar{\varepsilon}}{2}, t_{1}+\bar{\varepsilon}\right)$ and $\tau_{2} \in\left(\frac{t_{1}+t_{2}}{2}, \frac{t_{1}+t_{2}}{2}+\frac{\bar{\varepsilon}}{2}\right)$ such that

$$
\begin{gathered}
e^{-\lambda\left(t_{1}+\bar{\varepsilon}\right)}-e^{-\lambda\left(t_{1}+\frac{\bar{\varepsilon}}{2}\right)}=\frac{\bar{\varepsilon}}{2}(-\lambda) e^{-\lambda \tau_{1}}, \\
e^{-\lambda\left(\frac{t_{1}+t_{2}}{2}+\frac{\bar{\varepsilon}}{2}\right)}-e^{-\lambda \frac{t_{1}+t_{2}}{2}}=\frac{\bar{\varepsilon}}{2}(-\lambda) e^{-\lambda \tau_{2}} .
\end{gathered}
$$

Thus, $\Delta\left(t_{1}, t_{2}\right)-\Delta\left(t_{1}, t_{1}+\bar{\varepsilon}\right)-\Delta\left(t_{1}+\bar{\varepsilon}, t_{2}\right)=\bar{\varepsilon} \lambda\left[e^{-\lambda \tau_{1}}-e^{-\lambda \tau_{2}}\right]$. As the function $r(t)$ is strictly decreasing on $\mathbb{R}$ and $\tau_{1}<\tau_{2}$, one gets $e^{-\lambda \tau_{1}}-e^{-\lambda \tau_{2}}>0$; hence the inequality (3.23) is proved.

Lemma 3.5. Let there be given $t_{1}, t_{2} \in\left[t_{0}, T\right], t_{1}<t_{2}$, and $\xi>0$. Suppose that $\left(\widetilde{x}^{\xi}, \widetilde{u}^{\xi}\right)$ and $\left(\check{x}^{\xi}, \check{u}^{\xi}\right)$ are feasible processes for $\left(F P_{3 a}\right)$ with $\widetilde{x}_{1}^{\xi}(t)=\xi$ for all $t \in\left[t_{1}, t_{2}\right]$ and

$$
\check{x}_{1}^{\xi}(t)= \begin{cases}\xi-a\left(t-t_{1}\right), & t \in\left[t_{1}, \check{t}\right] \\ \xi+a\left(t-t_{2}\right), & t \in\left(\check{t}, t_{2}\right],\end{cases}
$$

where $\check{t}:=2^{-1}\left(t_{1}+t_{2}\right)$. Then one has

$$
\left.J\left(\check{x}^{\xi}, \check{u}^{\xi}\right)\right|_{\left[t_{1}, t_{2}\right]}-\left.J\left(\widetilde{x}^{\xi}, \widetilde{u}^{\xi}\right)\right|_{\left[t_{1}, t_{2}\right]}=\frac{1}{\lambda}\left(\frac{a}{\lambda}-1\right) \Delta\left(t_{1}, t_{2}\right),
$$

with $\left.J(x, u)\right|_{\left[t_{1}, t_{2}\right]}$ and $\Delta\left(t_{1}, t_{2}\right)$ being defined respectively by (3.17) and (3.20). Besides, the strict inequality $\left.J\left(\check{x}^{\xi}, \check{u}^{\xi}\right)\right|_{\left[t_{1}, t_{2}\right]}>\left.J\left(\widetilde{x}^{\xi}, \widetilde{u}^{\xi}\right)\right|_{\left[t_{1}, t_{2}\right]}$ is valid.

Proof. The proof is similar to that of Lemma 3.2 .

Proposition 3.6. The situation where $\bar{x}_{1}(t)=-1$ for all $t$ from a subsegment $\left[t_{1}, t_{2}\right]$ of $\left[t_{0}, T\right]$ with $t_{1}<t_{2}$ cannot happen.

Proof. Since $(\bar{x}, \bar{u})$ is a $W^{1,1}$ local minimizer of $\left(F P_{3 a}\right)$, by Definition 2.1 there exists $\delta>0$ such that the process $(\bar{x}, \bar{u})$ minimizes the quantity $g\left(x\left(t_{0}\right), x(T)\right)=x_{2}(T)$ over all feasible processes $(x, u)$ of $\left(F P_{3 a}\right)$ with $\|\bar{x}-x\|_{W^{1,1}\left(\left[t_{0}, T\right] ; \mathbb{R}^{2}\right)} \leq \delta$.

To prove our assertion, suppose on the contrary that there are $t_{1}, t_{2}$ with $t_{0} \leq t_{1}<t_{2} \leq T$ such that $\bar{x}_{1}(t)=-1$ for all $t \in\left[t_{1}, t_{2}\right]$. Fixing a number $\varepsilon \in\left(0, t_{2}-t_{1}\right)$, we consider the pair of functions $\left(\hat{x}^{\varepsilon}, \hat{u}^{\varepsilon}\right)$, where

$$
\hat{x}_{1}^{\varepsilon}(t):= \begin{cases}\bar{x}_{1}(t), & t \in\left[t_{0}, t_{1}\right) \cup\left(t_{1}+\varepsilon, T\right] \\ -1+a\left(t-t_{1}\right), & t \in\left[t_{1}, t_{1}+2^{-1} \varepsilon\right] \\ -1-a\left(t-t_{1}-\varepsilon\right), & t \in\left(t_{1}+2^{-1} \varepsilon, t_{1}+\varepsilon\right]\end{cases}
$$


and $\hat{u}^{\varepsilon}(t):=-a^{-1} \frac{d \hat{x}_{1}^{\varepsilon}(t)}{d t}$ for almost all $t \in\left[t_{0}, T\right]$. Clearly, $\left(\hat{x}^{\varepsilon}, \hat{u}^{\varepsilon}\right)$ is a feasible process of $\left(F P_{3 a}\right)$. By (3.9), (3.17), and the definition of $\hat{x}_{1}^{\varepsilon}($.$) , we have$

$$
\bar{x}_{2}(T)-\hat{x}_{2}^{\varepsilon}(T)=J(\bar{x}, \bar{u})_{\mid\left[t_{1}, t_{1}+\varepsilon\right]}-J\left(\hat{x}^{\varepsilon}, \hat{u}^{\varepsilon}\right)_{\mid\left[t_{1}, t_{1}+\varepsilon\right]} .
$$

Besides, it follows from Lemma 3.3 and the constructions of $\bar{x}$ and $\hat{x}^{\varepsilon}$ on $\left[t_{1}, t_{1}+\varepsilon\right]$ that

$$
J(\bar{x}, \bar{u})_{\mid\left[t_{1}, t_{1}+\varepsilon\right]}-J\left(\hat{x}^{\varepsilon}, \hat{u}^{\varepsilon}\right)_{\mid\left[t_{1}, t_{1}+\varepsilon\right]}>0 .
$$

Combining this with (3.26) yields $\bar{x}_{2}(T)>\hat{x}_{2}^{\varepsilon}(T)$, which contradicts the $W^{1,1}$ local optimality of $(\bar{x}, \bar{u})$, because $\left\|\bar{x}-\hat{x}^{\varepsilon}\right\|_{W^{1,1}\left(\left[t_{0}, T\right] ; \mathbb{R}^{2}\right)} \leq \delta$ for $\varepsilon>0$ small enough.

The following two propositions are crucial for describing the behavior of the local solutions of $\left(F P_{3 a}\right)$.

Proposition 3.7. One must have $\bar{x}_{1}(t)>-1$ for all $t \in\left(t_{0}, T\right)$.

Proof. By our standing assumption, $(\bar{x}, \bar{u})$ is a $W^{1,1}$ a local minimizer for $\left(F P_{3 a}\right)$. Let $\delta>0$ be chosen as in the proof of Proposition [3.6. If the assertion is false, there would exist $\check{t} \in\left(t_{0}, T\right)$ with $\bar{x}_{1}(\check{t})=-1$.

If there are $\varepsilon_{1}>0$ and $\varepsilon_{2}>0$ such that $\bar{x}_{1}(t)>-1$ for all $t \in\left(\check{t}-\varepsilon_{1}, \check{t}\right) \cup\left(\check{t}, \check{t}+\varepsilon_{2}\right)$. Then, thanks to the continuity of $\bar{x}_{1}($.$) , by shrinking \varepsilon_{1}>0$ and $\varepsilon_{2}>0$ (if necessary) one may assume that $\bar{x}_{1}(t) \in(-1,1)$ for all $t \in\left(\check{t}-\varepsilon_{1}, \check{t}\right) \cup\left(\check{t}, \check{t}+\varepsilon_{2}\right)$. Then, since the curve $\bar{x}_{1}($. cannot have more than one turning on the interval $\left(\check{t}-\varepsilon_{1}, \check{t}\right)$ (resp., on the interval $\left(\check{t}, \check{t}+\varepsilon_{2}\right)$ ) by the observation given at the beginning of the proof of Proposition 3.1. So, replacing $\varepsilon_{1}$ (resp., $\varepsilon_{2}$ ) by a smaller positive number, one may assume that

$$
\bar{x}_{1}(t)= \begin{cases}-1-a(t-\check{t}), & t \in\left[\check{t}-\varepsilon_{1}, \check{t}\right] \\ -1+a(t-\check{t}), & t \in\left(\check{t}, \check{t}+\varepsilon_{2}\right] .\end{cases}
$$

To get a contradiction, we can apply the construction given in Lemma 3.5. Namely, choose $\varepsilon>0$ as small as $\varepsilon<\min \left\{\varepsilon_{1}, \varepsilon_{2}\right\}$ and define a feasible process $\left(\widetilde{x}^{\varepsilon}, \widetilde{u}^{\varepsilon}\right)$ for $\left(F P_{3 a}\right)$ by setting

$$
\widetilde{u}^{\varepsilon}(t)= \begin{cases}0, & t \in[\check{t}-\varepsilon, \check{t}+\varepsilon] \\ \bar{u}(t), & t \in\left[t_{0}, \check{t}-\varepsilon\right) \cup(\check{t}+\varepsilon, T]\end{cases}
$$

and

$$
\widetilde{x}^{\varepsilon}(t)= \begin{cases}\bar{x}_{1}(\check{t}-\varepsilon), & t \in[\check{t}-\varepsilon, \check{t}+\varepsilon] \\ \bar{x}(t), & t \in\left[t_{0}, \check{t}-\varepsilon\right) \cup(\check{t}+\varepsilon, T] .\end{cases}
$$

Then, by Lemma 3.5 one has $J(\bar{x}, \bar{u})>J\left(\widetilde{x}^{\varepsilon}, \widetilde{u}^{\varepsilon}\right)$. This contradicts the $W^{1,1}$ local optimality of $(\bar{x}, \bar{u})$, because $\left\|\bar{x}-\hat{x}^{\varepsilon}\right\|_{W^{1,1}\left(\left[t_{0}, T\right] ; \mathbb{R}^{2}\right)} \leq \delta$ for $\varepsilon>0$ small enough. 
Since one cannot find $\varepsilon_{1}>0$ and $\varepsilon_{2}>0$ such that the strict inequality $\bar{x}_{1}(t)>-1$ holds for all $t \in\left(\check{t}-\varepsilon_{1}, \check{t}\right) \cup\left(\check{t}, \check{t}+\varepsilon_{2}\right)$, there must exist a sequence $\left\{t_{k}\right\}$ in $\left(t_{0}, T\right)$ converging to $\check{t}$ such that either $t_{k}<\check{t}$ for all $k$ or $t_{k}>\check{t}$ for all $k$, and $\bar{x}_{1}\left(t_{k}\right)=-1$ for each $k$. It suffices to consider the case $t_{k}<\check{t}$ for all $k$, as the other case can be treated similarly. By considering a subsequence (if necessary), we may assume that $t_{k}<t_{k+1}$ for all $k$.

Choose $\bar{k}$ as large as

$$
\check{t}-t_{\bar{k}}<\min \left\{2 \delta a^{-1}, 4 a^{-1}\right\} .
$$

This choice of $\bar{k}$ guarantees that $\bar{x}_{1}(t)<1$ for every $t \in\left[t_{\bar{k}}, \check{t}\right]$. Indeed, otherwise there is some $\alpha \in\left(t_{\bar{k}}, \check{t}\right)$ with $\bar{x}_{1}(\alpha)=1$. Setting

$$
\alpha_{1}=\min \left\{t \in\left[t_{\bar{k}}, \alpha\right]: \bar{x}_{1}(t)=1\right\}, \quad \alpha_{2}=\max \left\{t \in[\alpha, \check{t}]: \bar{x}_{1}(t)=1\right\},
$$

one has $\alpha_{1} \leq \alpha_{2},\left[\alpha_{1}, \alpha_{2}\right] \subset\left[t_{\bar{k}}, \check{t}\right]$, and $\bar{x}_{1}(t) \in(-1,1)$ for all $t \in\left(t_{\bar{k}}, \alpha_{1}\right) \cup\left(\alpha_{2}, \check{t}\right)$. Then, by assertion S1 of Proposition 3.1, one has $\alpha_{1}-t_{\bar{k}}=2 a^{-1}$. Similarly, by assertion S2 in that proposition, one has $\check{t}-\alpha_{2}=2 a^{-1}$. So, one gets $\check{t}-t_{\bar{k}} \geq 4 a^{-1}$, which comes in conflict with (3.30).

By Proposition [3.6, one cannot have $\bar{x}_{1}(t)=-1$ for all $t \in\left[t_{\bar{k}}, t_{\bar{k}+1}\right]$. Thus, there is some $\tau \in\left(t_{\bar{k}}, t_{\bar{k}+1}\right)$ with $\bar{x}_{1}(\tau)>-1$. Setting

$$
\tau_{1}=\max \left\{t \in\left[t_{\bar{k}}, \tau\right]: \bar{x}_{1}(t)=-1\right\}, \tau_{2}=\min \left\{t \in\left[\tau, t_{\bar{k}+1}\right]: \bar{x}_{1}(t)=-1\right\},
$$

one has $\tau_{1}<\tau_{2},\left[\tau_{1}, \tau_{2}\right] \subset\left[t_{\bar{k}}, t_{\bar{k}+1}\right]$, and $\bar{x}_{1}(t) \in(-1,1)$ for all $t \in\left(\tau_{1}, \tau_{2}\right)$. Hence, replacing $t_{\bar{k}}$ (resp., $t_{\bar{k}+1}$ ) by $\tau_{1}$ (resp., $\tau_{2}$ ), one sees that all the above-described properties of the sequence $\left\{t_{k}\right\}$ remain and, in addition,

$$
\bar{x}_{1}(t) \in(-1,1), \quad \forall t \in\left(t_{\bar{k}}, t_{\bar{k}+1}\right) .
$$

Let $F:=\left\{t \in\left[t_{\bar{k}}, \check{t}\right]: \bar{x}_{1}(t)=-1\right\}$ and $E:=\left[t_{\bar{k}}, \check{t}\right] \backslash F$. Since $F$ is a closed subset of $\mathbb{R}$ and $E=\left(t_{\bar{k}}, \check{t}\right) \backslash F, E$ is an open subset of $\mathbb{R}$. So, $E$ is the union of a countable family of disjoint open intervals (see [16, Proposition 9, p. 17]). Since $t_{k} \notin E$ for all $k$, we have a representation $E=\bigcup_{i=1}^{\infty} E_{i}$, where the intervals $E_{i}=\left(\tau_{1}^{(i)}, \tau_{2}^{(i)}\right), i \in \mathbb{N}$, are nonempty and disjoint. Thanks to (3.31), one may suppose that $E_{1}=\left(\tau_{1}^{(1)}, \tau_{2}^{(1)}\right)=\left(t_{\bar{k}}, t_{\bar{k}+1}\right)$. Note also that, for any $i \in \mathbb{N}, \bar{x}_{1}(t) \in(-1,1)$ for all $t \in E_{i}$. Since $\bar{x}_{1}\left(\tau_{1}^{(i)}\right)=\bar{x}_{1}\left(\tau_{2}^{(i)}\right)=-1$, by assertion S3 of Proposition 3.1 one gets

$$
\bar{x}_{1}(t)= \begin{cases}-1+a\left(t-\tau_{1}^{(i)}\right), & t \in\left[\tau_{1}^{(i)}, 2^{-1}\left(\tau_{1}^{(i)}+\tau_{2}^{(i)}\right)\right] \\ -1-a\left(t-\tau_{2}^{(i)}\right), & t \in\left(2^{-1}\left(\tau_{1}^{(i)}+\tau_{2}^{(i)}\right), \tau_{2}^{(i)}\right] .\end{cases}
$$

If the set $F_{1}:=F \backslash\left\{t_{\bar{k}}\right\}$ has an isolated point in the induced topology of $\left[t_{\bar{k}}, \check{t}\right]$, says, $\bar{t}$. Then, one must have $\bar{t} \in\left[t_{\bar{k}+1}, \check{t}\right)$. So, there exists $\varepsilon>0$ such that $(\bar{t}-\varepsilon, \bar{t}+\varepsilon) \subset\left(t_{\bar{k}}, \check{t}\right)$ 
and $\bar{x}_{1}(t) \in(-1,1)$ for all $t \in(\bar{t}-\varepsilon, \bar{t}) \cup(\bar{t}, \bar{t}+\varepsilon)$. Applying the construction given in the first part of this proof, we find a feasible process $\left(\widetilde{x}^{\varepsilon}, \widetilde{u}^{\varepsilon}\right)$ for $\left(F P_{3 a}\right)$ with the property $J(\bar{x}, \bar{u})>J\left(\widetilde{x}^{\varepsilon}, \widetilde{u}^{\varepsilon}\right)$. This contradicts the $W^{1,1}$ local optimality of $(\bar{x}, \bar{u})$, because (3.30) assures that $\left\|\bar{x}-\hat{x}^{\varepsilon}\right\|_{W^{1,1}\left(\left[t_{0}, T\right] ; \mathbb{R}^{2}\right)} \leq \delta$.

Now, suppose that every point in the compact set $F_{1}$ is a limit point of this set in the induced topology of $\left[t_{\bar{k}}, \check{t}\right]$. Then, if the Lebesgue measure $\mu_{L}\left(F_{1}\right)$ of $F_{1}$ is null, then the structure of $F_{1}$ is similar to that of the Cantor set 1 , constructed from the segment $\left[t_{\bar{k}+1}, \check{t}\right] \subset \mathbb{R}$. If $\mu_{L}\left(F_{1}\right)>0$, the structure of $F_{1}$ is similar to that of a fat Cantor set, which is also called a Smith-Volterra-Cantor set2.

Putting

$$
\widetilde{u}(t)= \begin{cases}0, & t \in\left[t_{\bar{k}}, \check{t}\right] \\ \bar{u}(t), & t \in\left[t_{0}, t_{\bar{k}}\right) \cup(\check{t}, T]\end{cases}
$$

and

$$
\widetilde{x}_{1}(t)= \begin{cases}-1, & t \in\left[t_{\bar{k}}, \check{t}\right] \\ \bar{x}_{1}(t), & t \in\left[t_{0}, t_{\bar{k}}\right) \cup(\check{t}, T],\end{cases}
$$

we see that $(\widetilde{x}, \widetilde{u})$ is a feasible process for $\left(F P_{3 a}\right)$. Similarly, define

$$
u(t)= \begin{cases}-1, & t \in\left[t_{\bar{k}+1}, 2^{-1}\left(t_{\bar{k}+1}+\check{t}\right)\right] \\ 1, & t \in\left(2^{-1}\left(t_{\bar{k}+1}+\check{t}\right), \check{t}\right] \\ \bar{u}(t), & t \in\left[t_{0}, t_{\bar{k}+1}\right) \cup(\check{t}, T]\end{cases}
$$

and

$$
x_{1}(t)= \begin{cases}-1+a\left(t-t_{\bar{k}+1}\right), & t \in\left[t_{\bar{k}+1}, 2^{-1}\left(t_{\bar{k}}+\check{t}\right)\right] \\ -1-a(t-\check{t}), & t \in\left(2^{-1}\left(t_{\bar{k}+1}+\check{t}\right), \check{t}\right] \\ \bar{x}_{1}(t), & t \in\left[t_{0}, t_{\bar{k}+1}\right) \cup(\check{t}, T],\end{cases}
$$

and observe that $(x, u)$ is a feasible process for $\left(F P_{3 a}\right)$. Using (3.30), it is easy to verify that $\|x-\bar{x}\|_{W^{1,1}\left(\left[t_{0}, T\right] ; \mathbb{R}^{2}\right)} \leq \delta$. Thus, if it can be shown that

$$
J(x, u)<J(\bar{x}, \bar{u})
$$

then we get a contradiction to the $W^{1,1}$ local optimality of $(\bar{x}, \bar{u})$. Hence, the proof of the lemma will be completed.

By (3.33)-(3.36) and Lemma 3.3 , one has $J(\widetilde{x}, \widetilde{u})-J(x, u)=\left.J(\widetilde{x}, \widetilde{u})\right|_{\left[t_{\bar{k}}, \tilde{t}\right]}-\left.J(x, u)\right|_{\left[t_{\bar{k}}, \tilde{t}\right]}$. Therefore,

$$
J(\widetilde{x}, \widetilde{u})-J(x, u)=\frac{1}{\lambda}\left(\frac{a}{\lambda}-1\right)\left[\Delta\left(t_{\bar{k}}, t_{\bar{k}+1}\right)+\Delta\left(t_{\bar{k}+1}, \check{t}\right)\right],
$$

\footnotetext{
${ }^{1}$ https://en.wikipedia.org/wiki/Cantor_set.

${ }^{2}$ https://en.wikipedia.org/wiki/Smith-Volterra-Cantor_set.
} 
where $\Delta\left(t_{1}, t_{2}\right)$, for any $t_{1}, t_{2}$ with $t_{1}<t_{2}$, is given by (3.20). In addition, using (3.33), (3.34), the decomposition $\left[t_{\bar{k}+1}, \check{t}\right]=\left(\bigcup_{i=2}^{\infty} E_{i}\right) \cup F_{1}$, and the sum rule [14, Theorem 1', p. 297] and the decomposition formula [14, Theorem 4, p. 298] for the Lebesgue integrals, one gets

$$
\begin{aligned}
J(\bar{x}, \bar{u})-J(\widetilde{x}, \widetilde{u}) & =\left.J(\bar{x}, \bar{u})\right|_{\left[t_{\bar{k}}, \tilde{t}\right]}-\left.J(\widetilde{x}, \widetilde{u})\right|_{\left[t_{\bar{k}}, \tilde{t}\right]} \\
& =\int_{\left[t_{\bar{k}}, \tilde{t}\right]}\left[-e^{-\lambda t}\left(\left[\bar{x}_{1}(t)+\bar{u}(t)\right]-\left[\widetilde{x}_{1}(t)+\widetilde{u}(t)\right]\right)\right] d t \\
& =\sum_{i=2}^{\infty} \int_{E_{i}}\left[-e^{-\lambda t}\left(\left[\bar{x}_{1}(t)+\bar{u}(t)\right]-\left[\widetilde{x}_{1}(t)+\widetilde{u}(t)\right]\right)\right] d t \\
& +\int_{F_{1}}\left[-e^{-\lambda t}\left(\left[\bar{x}_{1}(t)+\bar{u}(t)\right]-\left[\widetilde{x}_{1}(t)+\widetilde{u}(t)\right]\right)\right] d t .
\end{aligned}
$$

Hence, it holds that

$$
J(\bar{x}, \bar{u})-J(\widetilde{x}, \widetilde{u})=-\frac{1}{\lambda}\left(\frac{a}{\lambda}-1\right) \sum_{i=2}^{\infty} \Delta\left(\tau_{1}^{(i)}, \tau_{2}^{(i)}\right)+I,
$$

where $I:=\int_{F_{1}}\left[-e^{-\lambda t}\left(\left[\bar{x}_{1}(t)+\bar{u}(t)\right]-\left[\widetilde{x}_{1}(t)+\widetilde{u}(t)\right]\right)\right] d t$. Given any $t \in F_{1}$, we observe that $\bar{x}_{1}(t)=\widetilde{x}_{1}(t)=-1$ and $\widetilde{u}(t)=0$. Since every point in $F_{1}$ is a limit point of this set in the induced topology of $\left[t_{\bar{k}}, \check{t}\right]$, we can find a sequence $\left\{\xi_{j}^{t}\right\}$ in $F_{1}$ satisfying $\lim _{j \rightarrow \infty} \xi_{j}^{t}=t$. As the derivative $\bar{x}_{1}(t)$ exists a.e. on $\left[t_{0}, T\right]$, it exists a.e. on $F_{1}$. In combination with the first differential equation in (3.11), this yields $\dot{\bar{x}}_{1}(t)=-a \bar{u}(t)$ a.e. $t \in F_{1}$. Since $\bar{x}_{1}(t)=-1$ for all $t \in F_{1}$, for a.e. $t \in F_{1}$ it holds that

$$
\bar{u}(t)=-\frac{1}{a} \dot{\bar{x}}_{1}(t)=-\frac{1}{a} \lim _{j \rightarrow \infty} \frac{\bar{x}_{1}\left(\xi_{j}^{t}\right)-\bar{x}_{1}(t)}{\xi_{j}^{t}-t}=0
$$

We have thus shown that $\left[\bar{x}_{1}(t)+\bar{u}(t)\right]-\left[\widetilde{x}_{1}(t)+\widetilde{u}(t)\right]=0$ for a.e. $t \in F_{1}$. This implies that $I=0$. Now, adding (3.38) (3.39), we get

$$
J(\bar{x}, \bar{u})-J(x, u)=\frac{1}{\lambda}\left(\frac{a}{\lambda}-1\right)\left[\Delta\left(t_{\bar{k}}, t_{\bar{k}+1}\right)+\Delta\left(t_{\bar{k}+1}, \check{t}\right)-\sum_{i=2}^{\infty} \Delta\left(\tau_{1}^{(i)}, \tau_{2}^{(i)}\right)\right] .
$$

We have

$$
\sum_{i=2}^{\infty} \Delta\left(\tau_{1}^{(i)}, \tau_{2}^{(i)}\right) \leq \Delta\left(t_{\bar{k}+1}, \check{t}\right) .
$$

To establish this inequality, we first show that

$$
\sum_{i=2}^{m} \Delta\left(\tau_{1}^{(i)}, \tau_{2}^{(i)}\right)<\Delta\left(t_{\bar{k}+1}, \check{t}\right)
$$


for any integer $m \geq 2$. Taking account of the fact that every point in $F_{1}$ is a limit point of this set in the induced topology of $\left[t_{\bar{k}}, \check{t}\right]$, by reordering the intervals $\left(\tau_{1}^{(i)}, \tau_{2}^{(i)}\right)$ for $i=2, \ldots, m$, we may assume that $t_{\bar{k}+1}<\tau_{1}^{(2)}<\tau_{2}^{(2)}<\tau_{1}^{(3)}<\tau_{2}^{(3)}<\cdots<\tau_{1}^{(m)}<\tau_{2}^{(m)}<\check{t}$. Then, by Lemma 3.4 and by induction, we have

$$
\begin{aligned}
\sum_{i=2}^{m} \Delta\left(\tau_{1}^{(i)}, \tau_{2}^{(i)}\right) & <\left[\Delta\left(t_{\bar{k}+1}, \tau_{1}^{(2)}\right)+\Delta\left(\tau_{1}^{(2)}, \tau_{2}^{(2)}\right)\right]+\sum_{i=3}^{m} \Delta\left(\tau_{1}^{(i)}, \tau_{2}^{(i)}\right) \\
& <\left[\Delta\left(t_{\bar{k}+1}, \tau_{2}^{(2)}\right)+\Delta\left(\tau_{2}^{(2)}, \tau_{1}^{(3)}\right)\right]+\sum_{i=3}^{m} \Delta\left(\tau_{1}^{(i)}, \tau_{2}^{(i)}\right) \\
& \vdots \\
& <\Delta\left(t_{\bar{k}+1}, \tau_{2}^{(m)}\right)+\Delta\left(\tau_{2}^{(m)}, \check{t}\right) \\
& <\Delta\left(t_{\bar{k}+1}, \check{t}\right) .
\end{aligned}
$$

Thus, (3.42) is valid. Since $\Delta\left(\tau_{1}^{(i)}, \tau_{2}^{(i)}\right)>0$ for all $i=2,3, \ldots$, the estimate (3.42) shows that the series $\sum_{i=2}^{\infty} \Delta\left(\tau_{1}^{(i)}, \tau_{2}^{(i)}\right)$ is convergent. Letting $m \rightarrow \infty$, from (3.42) one obtains (3.41). Since $\Delta\left(t_{\bar{k}}, t_{\bar{k}+1}\right)>0$, the equality (3.40) and the inequality (3.41) imply (3.37).

The proof is complete.

To continue, using the data set $\left\{a, \lambda, t_{0}, T, x_{0}\right\}$ of $\left(F P_{3 a}\right)$, we define $\rho=\frac{1}{\lambda} \ln \frac{a}{a-\lambda}>0$ and $\bar{t}=T-\rho$. Besides, for a given $x_{0} \in[-1,1]$, let

$$
\rho_{1}:=a^{-1}\left(1+x_{0}\right) \quad \text { and } \quad \rho_{2}:=a^{-1}\left(1-x_{0}\right)
$$

As $x_{0} \in[-1,1]$, one has $\rho_{1} \in\left[0,2 a^{-1}\right]$ and $\rho_{2} \in\left[0,2 a^{-1}\right]$. Moreover, since $\bar{x}_{1}(t)$ is a continuous function, $\mathcal{T}_{1}:=\left\{t \in\left[t_{0}, T\right]: \bar{x}_{1}(t)=1\right\}$ is a compact set (which may be empty). If $\mathcal{T}_{1}$ is nonempty, then we consider the numbers $\alpha_{1}:=\min \left\{t: t \in \mathcal{T}_{1}\right\}$ and $\alpha_{2}:=\max \left\{t: t \in \mathcal{T}_{1}\right\}$.

By Proposition [3.7, one of next four cases must occur.

Case 1: $\bar{x}_{1}(t)>-1$ for all $t \in\left[t_{0}, T\right]$. Then, condition (i) means that (3.14) and (3.15) are satisfied, while conditions (ii)-(iv) remain the same as those in Subsection 4.2 of Part 1 ([13]). So, the curve $\bar{x}_{1}(t)$ must have of one of the forms (a)-(c) depicted in Theorem 4.4 of Part 1 ([13]), where we let $\bar{x}_{1}(t)$ play the role of $\bar{x}(t)$. Of course, the condition $\bar{x}_{1}(t)>-1$ for all $t \in\left[t_{0}, T\right]$ must be satisfied. Note that the latter is equivalent to the requirement $\bar{x}_{1}(T)>-1$. With respect to the just mentioned three forms of $\bar{x}(t)$, we have the following three subcases.

Subcase 1a: $\bar{x}_{1}(t)$ is given by

$$
\bar{x}_{1}(t)=x_{0}-a\left(t-t_{0}\right), \quad t \in\left[t_{0}, T\right] .
$$


By statement (a) of Theorem 4.4 of Part 1 ([13]), this situation happens when $T-t_{0} \leq \rho$. By (3.44), condition $\bar{x}_{1}(T)>-1$ is equivalent to $T-t_{0}<\rho_{1}$. Therefore, if either $\rho<\rho_{1}$ and $T-t_{0} \leq \rho$, or $\rho \geq \rho_{1}$ and $T-t_{0}<\rho_{1}$, then $\bar{x}_{1}(t)$ is given by (3.44).

Subcase $1 b: \bar{x}_{1}(t)$ is given by

$$
\bar{x}_{1}(t)= \begin{cases}x_{0}+a\left(t-t_{0}\right), & t \in\left[t_{0}, \bar{t}\right] \\ x_{0}-a\left(t+t_{0}-2 \bar{t}\right), & t \in(\bar{t}, T] .\end{cases}
$$

Then, statement (b) of Theorem 4.4 of Part 1 ([13]) requires that $\rho<T-t_{0}<\rho+\rho_{2}$. By (3.45), the inequality $\bar{x}_{1}(T)>-1$ means $T-t_{0}>2 \rho-\rho_{1}$. Thus, if $\max \left\{\rho ; 2 \rho-\rho_{1}\right\}<$ $T-t_{0}<\rho+\rho_{2}$, then $\bar{x}_{1}(t)$ is given by (3.45).

Subcase $1 c: \bar{x}_{1}(t)$ is given by

$$
\bar{x}_{1}(t)= \begin{cases}x_{0}+a\left(t-t_{0}\right), & t \in\left[t_{0}, t_{0}+\rho_{2}\right] \\ 1-a\left(t-t_{0}-\rho_{2}\right), & t \in\left(t_{0}+\rho_{2}, T\right] .\end{cases}
$$

Since $\alpha_{1}=t_{0}+a^{-1}\left(1-x_{0}\right)=t_{0}+\rho_{2}$, this situation is in full agreement with the one in assertion (c) of Theorem 4.4 of Part 1 ([13]). Here, one must have $T-t_{0} \geq \rho+\rho_{2}$. By (3.46), the inequality $\bar{x}_{1}(T)>-1$ means $T-t_{0}<a^{-1}\left(3-x_{0}\right)$. Thus, this situation occurs if $\rho+\rho_{2} \leq T-t_{0}<a^{-1}\left(3-x_{0}\right)$.

Case 2: $\bar{x}_{1}\left(t_{0}\right)=-1$ and $\bar{x}_{1}(t)>-1$ for all $t \in\left(t_{0}, T\right]$. Let $\bar{\varepsilon}>0$ be such that $t_{0}+\bar{\varepsilon}<T$. For any $k \in \mathbb{N}$ with $k^{-1} \in(0, \bar{\varepsilon})$, by the comments before Propositions 4.1 and by Proposition 4.2 of Part 1 ([13]) we can assert that the restriction of $(\bar{x}, \bar{u})$ on $\left[t_{0}+k^{-1}, T\right]$ is a $W^{1,1}$ local minimizer for the Mayer problem obtained from $\left(F P_{3 a}\right)$ by replacing $t_{0}$ with $t_{0}+k^{-1}$. Since $\bar{x}_{1}(t)>-1$ for all $t \in\left[t_{0}+k^{-1}, T\right]$, repeating the arguments already used in Case 1 yields a formula for $\bar{x}_{1}(t)$ on $\left[t_{0}+k^{-1}, T\right]$. With $\rho_{1}(k):=a^{-1}\left[1+\bar{x}_{1}\left(t_{0}+k^{-1}\right)\right]$ and $\rho_{2}(k):=a^{-1}\left[1-\bar{x}_{1}\left(t_{0}+k^{-1}\right)\right]$, for every $k \in \mathbb{N}$ we see that the function $\bar{x}_{1}(t)$ on $\left[t_{0}+k^{-1}, T\right]$ must belong to one of the following three categories, which correspond to the three forms of the function $\bar{x}_{1}(t)$ in Case 1 .

(C1) $\bar{x}_{1}(t)$ is given by

$$
\bar{x}_{1}(t)=\bar{x}_{1}\left(t_{0}+k^{-1}\right)-a\left(t-t_{0}-k^{-1}\right), \quad t \in\left[t_{0}+k^{-1}, T\right]
$$

provided that $\rho<\rho_{1}(k)$ and $T-t_{0}-k^{-1} \leq \rho$, or $\rho \geq \rho_{1}(k)$ and $T-t_{0}-k^{-1}<\rho_{1}(k)$.

(C2) $\bar{x}_{1}(t)$ is given by

$$
\bar{x}_{1}(t)= \begin{cases}\bar{x}_{1}\left(t_{0}+k^{-1}\right)+a\left(t-t_{0}-k^{-1}\right), & t \in\left[t_{0}, \bar{t}\right] \\ \bar{x}_{1}\left(t_{0}+k^{-1}\right)-a\left(t+t_{0}+k^{-1}-2 \bar{t}\right), & t \in(\bar{t}, T],\end{cases}
$$

provided that $\max \left\{\rho ; 2 \rho-\rho_{1}(k)\right\}<T-t_{0}-k^{-1}<\rho+\rho_{2}(k)$. 
(C3) $\bar{x}_{1}(t)$ is given by

$$
\bar{x}_{1}(t)= \begin{cases}\bar{x}_{1}\left(t_{0}+k^{-1}\right)+a\left(t-t_{0}-k^{-1}\right), & t \in\left[t_{0}, t_{0}+\rho_{2}(k)\right] \\ 1-a\left(t-t_{0}-k^{-1}-\rho_{2}(k)\right), & t \in\left(t_{0}+\rho_{2}(k), T\right],\end{cases}
$$

provided that $\rho+\rho_{2}(k) \leq T-t_{0}-k^{-1}<a^{-1}\left[3-\bar{x}_{1}\left(t_{0}+k^{-1}\right)\right]$.

By the Dirichlet principle, there exist an infinite number of indexes $k$ with $k^{-1} \in(0, \bar{\varepsilon})$ such that the formula for $\bar{x}_{1}(t)$ is given in the category $\mathrm{C} 1$ (resp., C2, or C3). By considering a subsequence if necessary, we may assume that this happens for all $k$ with $k^{-1} \in(0, \bar{\varepsilon})$.

If the first situation occurs, then by letting $k \rightarrow \infty$ we have $\bar{x}_{1}(t)=-1-a\left(t-t_{0}\right)$ for all $t \in\left[t_{0}, T\right]$. This is impossible since the requirement $\bar{x}_{1}(t)>-1$ for all $t \in\left(t_{0}, T\right]$ is violated.

If the second situation occurs, then by letting $k \rightarrow \infty$ we have

$$
\bar{x}_{1}(t)= \begin{cases}-1+a\left(t-t_{0}\right), & t \in\left[t_{0}, \bar{t}\right] \\ -1-a\left(t+t_{0}-2 \bar{t}\right), & t \in(\bar{t}, T]\end{cases}
$$

provided that $2 \rho \leq T-t_{0} \leq \rho+2 a^{-1}$. Since $\bar{x}_{1}(t)>-1$ for all $t \in\left(t_{0}, T\right]$, especially $\bar{x}_{1}(T)>-1$, one must have $2 \rho<T-t_{0}$.

If the last situation occurs, then $\bar{x}_{1}(t)$ is given by

$$
\bar{x}_{1}(t)= \begin{cases}-1+a\left(t-t_{0}\right), & t \in\left[t_{0}, t_{0}+2 a^{-1}\right] \\ 1-a\left(t-t_{0}-2 a^{-1}\right), & t \in\left(t_{0}+2 a^{-1}, T\right],\end{cases}
$$

provided that $\rho+2 a^{-1} \leq T-t_{0} \leq 4 a^{-1}$. Having in mind that $\bar{x}_{1}(T)>-1$, one must have the strict inequality $T-t_{0}<4 a^{-1}$.

Since the first situation cannot happen and since $\bar{t}=t_{0}+2 a^{-1}$ when $T-t_{0}=\rho+2 a^{-1}$, our results in this case can be summarized as follows.

Subcase 2a: $\bar{x}_{1}(t)$ is given by (3.47), provided that $2 \rho<T-t_{0}<\rho+2 a^{-1}$.

Subcase 2b: $\bar{x}_{1}(t)$ is given by (3.48), provided that $\rho+2 a^{-1} \leq T-t_{0}<4 a^{-1}$.

Case 3: $\bar{x}_{1}(T)=-1$ and $\bar{x}_{1}(t)>-1$ for all $t \in\left[t_{0}, T\right)$. We split this case into two subcases.

Subcase 3a: $\mathcal{T}_{1}=\emptyset$. Then $\bar{x}_{1}(t) \in(-1,1)$ for all $t \in\left[t_{0}, T\right)$ and $\bar{x}_{1}(T)=-1$. By some arguments similar to those of the proof of Proposition 3.1, one can show that formula for $\bar{x}_{1}($.$) on \left[t_{0}, T\right]$ is one of the following two types:

$$
\bar{x}_{1}(t)=x_{0}-a\left(t-t_{0}\right), \quad t \in\left[t_{0}, T\right]
$$

and

$$
\bar{x}_{1}(t)= \begin{cases}x_{0}+a\left(t-t_{0}\right), & t \in\left[t_{0}, t_{\zeta}\right] \\ -1-a(t-T), & t \in\left(t_{\zeta}, T\right]\end{cases}
$$


with $t_{\zeta} \in\left(t_{0}, T\right)$.

If $\bar{x}_{1}($.$) is given by (3.49), then \bar{x}_{1}(T)=-1$ if and only if $T-t_{0}=\rho_{1}$. Since $x_{0} \in(-1,1]$, the latter yields $0<T-t_{0}=\rho_{1} \leq 2 a^{-1}$.

If $\bar{x}_{1}($.$) is of the form (3.50), then the equality \bar{x}_{1}(T)=-1$ implies that

$$
t_{\zeta}=2^{-1}\left[T+t_{0}-\rho_{1}\right]
$$

Since $t_{\zeta}>t_{0}$, one must have $T-t_{0}>\rho_{1}$. Meanwhile, by (3.50) and our standing assumption in the current subcase, $\bar{x}_{1}\left(t_{\zeta}\right)<1$. So, $T-t_{0}<\rho_{1}+2 \rho_{2}=a^{-1}\left(3-x_{0}\right)$. Combining this and the inequality $T-t_{0}>\rho_{1}$ yields $\rho_{1}<T-t_{0}<a^{-1}\left(3-x_{0}\right)$. Our results in this subcase can be summarized as follows:

- $\bar{x}_{1}($.$) is given by (3.49), provided that T-t_{0}=\rho_{1}$.

- $\bar{x}_{1}($.$) is given by (3.50), provided that \rho_{1}<T-t_{0}<a^{-1}\left(3-x_{0}\right)$.

Subcase 3b: $\mathcal{T}_{1} \neq \emptyset$. Then we have $t_{0} \leq \alpha_{1} \leq \alpha_{2}<T$. It follows from assertion S2 of Proposition 3.1 that $T-\alpha_{2}=2 a^{-1}$ and $\bar{x}_{1}(t)=1-a\left(t-\alpha_{2}\right)$ for all $t \in\left[\alpha_{2}, T\right]$. Thus, we have $\alpha_{2}=T-2 a^{-1}$ and $\bar{x}_{1}(t)=1-a\left(t-T+2 a^{-1}\right)$ for all $t \in\left[T-2 a^{-1}, T\right]$.

If $\alpha_{1}<\alpha_{2}$, then $\bar{x}_{1}(t)=1$ for all $t \in\left[\alpha_{1}, \alpha_{2}\right]$. Indeed, suppose on the contrary that there exists $\bar{t} \in\left(\alpha_{1}, \alpha_{2}\right)$ satisfying $\bar{x}_{1}(\bar{t})<1$. Set

$$
\bar{\alpha}_{1}=\max \left\{t \in\left[\alpha_{1}, \bar{t}\right]: \bar{x}_{1}(t)=1\right\} \quad \text { and } \quad \bar{\alpha}_{2}=\min \left\{t \in\left[\bar{t}, \alpha_{2}\right]: \bar{x}_{1}(t)=1\right\} .
$$

Clearly, $\left[\bar{\alpha}_{1}, \bar{\alpha}_{2}\right] \subset\left[\alpha_{1}, \alpha_{2}\right] \subset\left[t_{0}, T\right)$ and $\bar{x}_{1}(t)<1$ for all $t \in\left(\bar{\alpha}_{1}, \bar{\alpha}_{2}\right)$. This and the condition $\bar{x}_{1}(t)>-1$ for all $t \in\left[t_{0}, T\right)$ imply that $\bar{x}_{1}(t) \in(-1,1)$ for all $t \in\left(\bar{\alpha}_{1}, \bar{\alpha}_{2}\right)$. So, by assertion $\mathrm{S} 4$ of Proposition 3.1, we obtain a contradiction. Our claim has been proved.

If $t_{0}<\alpha_{1}$, then $\bar{x}_{1}(t) \in(-1,1)$ for all $t \in\left[t_{0}, \alpha_{1}\right)$ and $\bar{x}_{1}\left(\alpha_{1}\right)=1$. Thus, repeating the arguments in the proof of assertion S1 of Proposition 3.1. we find that $\bar{x}_{1}(t)=x_{0}+a\left(t-t_{0}\right)$ for all $t \in\left[t_{0}, \alpha_{1}\right]$. As $\bar{x}_{1}\left(\alpha_{1}\right)=1$, we have $\alpha_{1}=t_{0}+\rho_{2}$. Consequently, the inequality as $T-t_{0} \geq\left(\alpha_{1}-t_{0}\right)+\left(T-\alpha_{2}\right)$ implies that $T-t_{0} \geq \rho_{2}+2 a^{-1}=a^{-1}\left(3-x_{0}\right)$. Our results in this subcase can be summarized as follows:

- $\bar{x}_{1}($.$) is given by$

$$
\bar{x}_{1}(t)= \begin{cases}x_{0}+a\left(t-t_{0}\right), & t \in\left[t_{0}, T-2 a^{-1}\right] \\ -1-a(t-T), & t \in\left(T-2 a^{-1}, T\right],\end{cases}
$$

provided that $T-t_{0}=a^{-1}\left(3-x_{0}\right)$.

- $\bar{x}_{1}($.$) is given by$

$$
\bar{x}_{1}(t)= \begin{cases}x_{0}+a\left(t-t_{0}\right), & t \in\left[t_{0}, t_{0}+\rho_{2}\right] \\ 1, & t \in\left(t_{0}+\rho_{2}, T-2 a^{-1}\right] \\ -1-a(t-T), & t \in\left(T-2 a^{-1}, T\right],\end{cases}
$$


provided that $T-t_{0}>a^{-1}\left(3-x_{0}\right)$.

Case 4: $\bar{x}_{1}\left(t_{0}\right)=\bar{x}_{1}(T)=-1$ and $\bar{x}_{1}(t)>-1$ for all $t \in\left(t_{0}, T\right)$.

Subcase $4 a: \mathcal{T}_{1}=\emptyset$. Then $\bar{x}_{1}(t) \in(-1,1)$ for all $t \in\left(t_{0}, T\right)$. Thus, by assertion S3 of Proposition 3.1 one has $T-t_{0}<4 a^{-1}$ and

$$
\bar{x}_{1}(t)= \begin{cases}-1+a\left(t-t_{0}\right), & t \in\left[t_{0}, 2^{-1}\left(t_{0}+T\right)\right] \\ -1-a(t-T), & t \in\left(2^{-1}\left(t_{0}+T\right), T\right] .\end{cases}
$$

Subcase $4 b: \mathcal{T}_{1} \neq \emptyset$. Then, the numbers $\alpha_{1}$ and $\alpha_{2}$ exist and $t_{0}<\alpha_{1} \leq \alpha_{2}<T$. It follows from statements S1 and S2 of Proposition 3.1 that $\alpha_{1}-t_{0}=T-\alpha_{2}=2 a^{-1}$ and $\bar{x}_{1}(t)=-1+a\left(t-t_{0}\right)$ for all $t \in\left[t_{0}, \alpha_{1}\right]$ and $\bar{x}_{1}(t)=1-a\left(t-\alpha_{2}\right)$ for all $t \in\left[\alpha_{2}, T\right]$. Thus, we have $\alpha_{1}=t_{0}+2 a^{-1}, \alpha_{2}=T-2 a^{-1}, \bar{x}_{1}(t)=-1+a\left(t-t_{0}\right)$ for all $t \in\left[t_{0}, t_{0}+2 a^{-1}\right]$, and $\bar{x}_{1}(t)=1-a\left(t-T+2 a^{-1}\right)$ for all $t \in\left[T-2 a^{-1}, T\right]$. Note that one must have $T-t_{0} \geq 4 a^{-1}$ in this subcase as $T-t_{0} \geq\left(\alpha_{1}-t_{0}\right)+\left(T-\alpha_{2}\right)$.

If $T-t_{0}>4 a^{-1}$, i.e., $\alpha_{1}<\alpha_{2}$, then by the result given in Subcase $3 \mathrm{~b}$ we have $\bar{x}_{1}(t)=1$ for all $t \in\left[t_{0}+2 a^{-1}, T-2 a^{-1}\right]$.

Our results in this case can be summarized as follows:

- $\bar{x}_{1}($.$) is given by$

$$
\bar{x}_{1}(t)= \begin{cases}-1+a\left(t-t_{0}\right), & t \in\left[t_{0}, 2^{-1}\left(t_{0}+T\right)\right] \\ -1-a(t-T), & t \in\left(2^{-1}\left(t_{0}+T\right), T\right],\end{cases}
$$

provided that $T-t_{0} \leq 4 a^{-1}$.

- $\bar{x}_{1}($.$) is given by$

$$
\bar{x}_{1}(t)= \begin{cases}-1+a\left(t-t_{0}\right), & t \in\left[t_{0}, t_{0}+2 a^{-1}\right] \\ 1, & t \in\left(t_{0}+2 a^{-1}, T-2 a^{-1}\right] \\ -1-a(t-T), & t \in\left(T-2 a^{-1}, T\right]\end{cases}
$$

provided that $T-t_{0}>4 a^{-1}$.

Now we turn our attention back to the original problem $\left(F P_{3}\right)$, which has a $W^{1,1}$ global solution (see Subsection [3.1). Using the given constants $a, \lambda$ with $a>\lambda>0$, we define $\rho=\frac{1}{\lambda} \ln \frac{a}{a-\lambda}>0$. This number $\rho$ is a characteristic constant of $\left(F P_{3}\right)$. From the analysis given in the present section we can obtain a complete synthesis of optimal processes. Due to the complexity of the possible trajectories, we prefer to present our results in six separate theorems. The first one treats the situation where $\rho \geq 2 a^{-1}$, while the other five deal with the situation where $\rho<2 a^{-1}$. 
Theorem 3.8. If $\rho \geq 2 a^{-1}$, then problem $\left(F P_{3}\right)$ has a unique local solution $(\bar{x}, \bar{u})$, which is a unique global solution, where $\bar{u}(t)=-a^{-1} \dot{\bar{x}}(t)$ for almost everywhere $t \in\left[t_{0}, T\right]$ and $\bar{x}(t)$ can be described as follows:

(a) If $T-t_{0} \leq a^{-1}\left(1+x_{0}\right)$, then

$$
\bar{x}(t)=x_{0}-a\left(t-t_{0}\right), \quad t \in\left[t_{0}, T\right] .
$$

(b) If $a^{-1}\left(1+x_{0}\right)<T-t_{0}<a^{-1}\left(3-x_{0}\right)$, then

$$
\bar{x}(t)= \begin{cases}x_{0}+a\left(t-t_{0}\right), & t \in\left[t_{0}, t_{\zeta}\right] \\ -1-a(t-T), & t \in\left(t_{\zeta}, T\right]\end{cases}
$$

with $t_{\zeta}:=2^{-1}\left[T+t_{0}-a^{-1}\left(1+x_{0}\right)\right]$.

(c) If $T-t_{0} \geq a^{-1}\left(3-x_{0}\right)$, then

$$
\bar{x}(t)=\left\{\begin{array}{lll}
x_{0}+a\left(t-t_{0}\right), & t \in\left[t_{0}, t_{0}+a^{-1}\left(1-x_{0}\right)\right] \\
1, & t \in\left(t_{0}+a^{-1}\left(1-x_{0}\right), T-2 a^{-1}\right] \\
-1-a(t-T), & t \in\left(T-2 a^{-1}, T\right] .
\end{array}\right.
$$

Proof. Suppose that $\rho \geq 2 a^{-1}$. Let $\rho_{1}, \rho_{2}$ be defined as in (3.43). Then, one has $\rho \geq \rho_{1}$, $2 \rho-\rho_{1} \geq \rho+\rho_{2}, \rho+\rho_{2} \geq 2 a^{-1}+\rho_{2}, 2 \rho \geq \rho+2 a^{-1}$, and $\rho+2 a^{-1} \geq 4 a^{-1}$. Thus, the analysis of Case 1 and Case 2 given before this theorem tells us that the situation in Subcase 1a happens when $T-t_{0}<\rho_{1}$, while the situations in Subcase 1b, Subcase 1c, and Case 2 cannot happen. Combining the results formulated in Subcase 1a, Case 3, Case 4, and noting that the function $\bar{x}_{1}(t)$ in $\left(F P_{3 a}\right)$ plays the role of $\bar{x}(t)$ in $\left(F P_{3}\right)$, we obtain the assertions of the theorem.

If $\rho<2 a^{-1}$, then the locally optimal processes of $\left(F P_{3}\right)$ depend greatly on the relative position of $x_{0}$ in the segment $[-1,1]$. In the forthcoming theorems, we distinguish five alternatives (one instance must occur, and any instance excludes others):

(i) $x_{0}=-1$;

(ii) $x_{0}>-1$ and $\rho<a^{-1}\left(1+x_{0}\right)<\rho+a^{-1}\left(1-x_{0}\right)$;

(iii) $x_{0}>-1$ and $a^{-1}\left(1+x_{0}\right)=\rho+a^{-1}\left(1-x_{0}\right)$;

(iv) $x_{0}>-1$ and $a^{-1}\left(1+x_{0}\right)>\rho+a^{-1}\left(1-x_{0}\right)$;

(v) $x_{0}>-1$ and $a^{-1}\left(1+x_{0}\right) \leq \rho$.

It is worthy to stress that to describe the possibilities (i)-(v) we have used just the parameters $a, \lambda$, and $x_{0}$. In each one of the situations $(\mathrm{i})-(\mathrm{v})$, the synthesis of the trajectories 
suspected for local minimizers of $\left(F P_{3}\right)$ is obtained by considering the position of the number $T-t_{0}>0$ on the half-line $[0,+\infty)$, which is divided into sections by the values $\rho, 2 \rho, \rho+2 a^{-1}$, $4 a^{-1}$, and other constants appeared in (i)-(v).

Theorem 3.9. If $\rho<2 a^{-1}$ and $x_{0}=-1$, then any local solution of problem $\left(F P_{3}\right)$ must have the form $(\bar{x}, \bar{u})$, where $\bar{u}(t)=-a^{-1} \dot{\bar{x}}(t)$ for almost everywhere $t \in\left[t_{0}, T\right]$ and $\bar{x}(t)$ is described as follows:

(a) If $T-t_{0} \leq 2 \rho$, then

$$
\bar{x}(t)= \begin{cases}-1+a\left(t-t_{0}\right), & t \in\left[t_{0}, 2^{-1}\left(t_{0}+T\right)\right] \\ -1-a(t-T), & t \in\left(2^{-1}\left(t_{0}+T\right), T\right]\end{cases}
$$

(b) If $2 \rho<T-t_{0}<\rho+2 a^{-1}$, then $\bar{x}(t)$ is given by either (3.52), or

$$
\bar{x}(t)= \begin{cases}-1+a\left(t-t_{0}\right), & t \in\left[t_{0}, \bar{t}\right] \\ -1-a\left(t+t_{0}-2 \bar{t}\right), & t \in(\bar{t}, T],\end{cases}
$$

where $\bar{t}=T-\rho$.

(c) If $\rho+2 a^{-1} \leq T-t_{0}<4 a^{-1}$, then $\bar{x}(t)$ is given by either (3.52), or

$$
\bar{x}(t)= \begin{cases}-1+a\left(t-t_{0}\right), & t \in\left[t_{0}, t_{0}+2 a^{-1}\right] \\ 1-a\left(t-t_{0}-2 a^{-1}\right), & t \in\left(t_{0}+2 a^{-1}, T\right] .\end{cases}
$$

(d) If $T-t_{0}=4 a^{-1}$, then $\bar{x}(t)$ is given by (3.52).

(e) If $T-t_{0}>4 a^{-1}$, then

$$
\bar{x}(t)= \begin{cases}-1+a\left(t-t_{0}\right), & t \in\left[t_{0}, t_{0}+2 a^{-1}\right] \\ 1, & t \in\left(t_{0}+2 a^{-1}, T-2 a^{-1}\right] \\ -1-a(t-T), & t \in\left(T-2 a^{-1}, T\right] .\end{cases}
$$

In the situations described in $(\mathrm{a}),(\mathrm{d})$, and $(\mathrm{e}),(\bar{x}, \bar{u})$ is a unique local solution of $\left(F P_{3}\right)$, which is also a unique global solution of the problem.

Proof. Suppose that $\rho<2 a^{-1}$ and $x_{0}=-1$. To obtain the assertions (a)-(e), it suffices to combine the results formulated in Case 2 and Case 4 , having in mind that $\bar{x}_{1}(t)$ in $\left(F P_{3 a}\right)$ plays the role of $\bar{x}(t)$ in $\left(F P_{3}\right)$. 
Theorem 3.10. If $\rho<2 a^{-1}, x_{0}>-1$, and $\rho<a^{-1}\left(1+x_{0}\right)<\rho+a^{-1}\left(1-x_{0}\right)$, then any local solution of problem $\left(F P_{3}\right)$ must have the form $(\bar{x}, \bar{u})$, where $\bar{u}(t)=-a^{-1} \dot{\bar{x}}(t)$ for almost everywhere $t \in\left[t_{0}, T\right]$ and $\bar{x}(t)$ is described as follows:

(a) If $T-t_{0} \leq \rho$, then $\bar{x}(t)$ is given by

$$
\bar{x}(t)=x_{0}-a\left(t-t_{0}\right), \quad t \in\left[t_{0}, T\right] .
$$

(b) If $\rho<T-t_{0}<a^{-1}\left(1+x_{0}\right)$, then

$$
\bar{x}(t)= \begin{cases}x_{0}+a\left(t-t_{0}\right), & t \in\left[t_{0}, \bar{t}\right] \\ x_{0}-a\left(t+t_{0}-2 \bar{t}\right), & t \in(\bar{t}, T],\end{cases}
$$

where $\bar{t}=T-\rho$.

(c) If $T-t_{0}=a^{-1}\left(1+x_{0}\right)$, then $\bar{x}(t)$ is given by either (3.53), or (3.54).

(d) If $a^{-1}\left(1+x_{0}\right)<T-t_{0}<\rho+a^{-1}\left(1-x_{0}\right)$, then $\bar{x}(t)$ is given by either (3.54), or

$$
\bar{x}(t)= \begin{cases}x_{0}+a\left(t-t_{0}\right), & t \in\left[t_{0}, t_{\zeta}\right] \\ -1-a(t-T), & t \in\left(t_{\zeta}, T\right]\end{cases}
$$

with $t_{\zeta}:=2^{-1}\left[T+t_{0}-a^{-1}\left(1+x_{0}\right)\right]$.

(e) If $\rho+a^{-1}\left(1-x_{0}\right) \leq T-t_{0}<a^{-1}\left(3-x_{0}\right)$, then $\bar{x}(t)$ is given by either (3.55), or

$$
\bar{x}(t)= \begin{cases}x_{0}+a\left(t-t_{0}\right), & t \in\left[t_{0}, t_{0}+a^{-1}\left(1-x_{0}\right)\right] \\ 1-a\left(t-t_{0}-a^{-1}\left(1-x_{0}\right)\right), & t \in\left(t_{0}+a^{-1}\left(1-x_{0}\right), T\right] .\end{cases}
$$

(f) If $T-t_{0}=a^{-1}\left(3-x_{0}\right)$, then

$$
\bar{x}(t)= \begin{cases}x_{0}+a\left(t-t_{0}\right), & t \in\left[t_{0}, T-2 a^{-1}\right] \\ -1-a(t-T), & t \in\left(T-2 a^{-1}, T\right] .\end{cases}
$$

(g) If $T-t_{0}>a^{-1}\left(3-x_{0}\right)$, then

$$
\bar{x}(t)= \begin{cases}x_{0}+a\left(t-t_{0}\right), & t \in\left[t_{0}, t_{0}+a^{-1}\left(1-x_{0}\right)\right] \\ 1, & t \in\left(t_{0}+a^{-1}\left(1-x_{0}\right), T-2 a^{-1}\right] \\ -1-a(t-T), & t \in\left(T-2 a^{-1}, T\right] .\end{cases}
$$

In the situations described in (a), (b), (f), and $(\mathrm{g}),(\bar{x}, \bar{u})$ is a unique local solution of $\left(F P_{3}\right)$, which is also a unique global solution of the problem. 
Proof. Suppose that $\rho<2 a^{-1}, x_{0}>-1$, and $\rho<a^{-1}\left(1+x_{0}\right)<\rho+a^{-1}\left(1-x_{0}\right)$. Let $\rho_{1}, \rho_{2}$ be defined as in (3.43). Then, combining the results formulated in Case 1 and Case 3, and noting that the function $\bar{x}_{1}(t)$ in $\left(F P_{3 a}\right)$ plays the role of $\bar{x}(t)$ in $\left(F P_{3}\right)$, we obtain the assertions (a) - (g).

Theorem 3.11. If $\rho<2 a^{-1}, x_{0}>-1$, and $a^{-1}\left(1+x_{0}\right)=\rho+a^{-1}\left(1-x_{0}\right)$, then any local solution of problem $\left(F P_{3}\right)$ must have the form $(\bar{x}, \bar{u})$, where $\bar{u}(t)=-a^{-1} \dot{\bar{x}}(t)$ for almost everywhere $t \in\left[t_{0}, T\right]$ and $\bar{x}(t)$ is described as follows:

(a) If $T-t_{0} \leq \rho$, then $\bar{x}(t)$ is given by (3.53).

(b) If $\rho<T-t_{0}<a^{-1}\left(1+x_{0}\right)$, then $\bar{x}(t)$ is given by (3.54).

(c) If $T-t_{0}=a^{-1}\left(1+x_{0}\right)$, then $\bar{x}(t)$ is given by either (3.53), or (3.56).

(d) If $a^{-1}\left(1+x_{0}\right)<T-t_{0}<a^{-1}\left(3-x_{0}\right)$, then $\bar{x}(t)$ is given by either (3.55), or (3.56).

(f) If $T-t_{0}=a^{-1}\left(3-x_{0}\right)$, then $\bar{x}(t)$ is given by (3.57).

(g) If $T-t_{0}>a^{-1}\left(3-x_{0}\right)$, then $\bar{x}(t)$ is given by (3.58).

In the situations described in (a), (b), (f), and $(\mathrm{g}),(\bar{x}, \bar{u})$ is a unique local solution of $\left(F P_{3}\right)$, which is also a unique global solution of the problem.

Proof. Suppose that $\rho<2 a^{-1}, x_{0}>-1$, and $a^{-1}\left(1+x_{0}\right)=\rho+a^{-1}\left(1-x_{0}\right)$. Then, combining the results formulated in Case 1 and Case 3, and noting that the function $\bar{x}_{1}(t)$ in $\left(F P_{3 a}\right)$ plays the role of $\bar{x}(t)$ in $\left(F P_{3}\right)$, we obtain the desired assertions.

Theorem 3.12. If $\rho<2 a^{-1}, x_{0}>-1$, and $a^{-1}\left(1+x_{0}\right)>\rho+a^{-1}\left(1-x_{0}\right)$, then any local solution of problem $\left(F P_{3}\right)$ must have the form $(\bar{x}, \bar{u})$, where $\bar{u}(t)=-a^{-1} \dot{\bar{x}}(t)$ for almost everywhere $t \in\left[t_{0}, T\right]$ and $\bar{x}(t)$ is described as follows:

(a) If $T-t_{0} \leq \rho$, then $\bar{x}(t)$ is given by (3.53).

(b) If $\rho<T-t_{0}<\rho+a^{-1}\left(1-x_{0}\right)$, then $\bar{x}(t)$ is given by (3.54).

(c) If $\rho+a^{-1}\left(1-x_{0}\right)<T-t_{0}<a^{-1}\left(1+x_{0}\right)$, then $\bar{x}(t)$ is given by (3.56)).

(d) If $T-t_{0}=a^{-1}\left(1+x_{0}\right)$, then $\bar{x}(t)$ is given by either (3.53), or (3.56).

(e) If $a^{-1}\left(1+x_{0}\right)<T-t_{0}<a^{-1}\left(3-x_{0}\right)$, then $\bar{x}(t)$ is given by either (3.55), or (3.56).

(f) If $T-t_{0}=a^{-1}\left(3-x_{0}\right)$, then $\bar{x}(t)$ is given by (3.57).

(g) If $T-t_{0}>a^{-1}\left(3-x_{0}\right)$, then $\bar{x}(t)$ is given by (3.58). 
In the situations described in (a), (b), (c), (f), and $(\mathrm{g}),(\bar{x}, \bar{u})$ is a unique local solution of $\left(F P_{3}\right)$, which is also a unique global solution of the problem.

Proof. Suppose that $\rho<2 a^{-1}, x_{0}>-1$, and $a^{-1}\left(1+x_{0}\right)>\rho+a^{-1}\left(1-x_{0}\right)$. Then, combining the results formulated in Case 1 and Case 3 , and noting that the function $\bar{x}_{1}(t)$ in $\left(F P_{3 a}\right)$ plays the role of $\bar{x}(t)$ in $\left(F P_{3}\right)$, we obtain the assertions of the theorem.

Theorem 3.13. If $\rho<2 a^{-1}, x_{0}>-1$, and $a^{-1}\left(1+x_{0}\right) \leq \rho$, then any local solution of problem $\left(F P_{3}\right)$ must have the form $(\bar{x}, \bar{u})$, where $\bar{u}(t)=-a^{-1} \dot{\bar{x}}(t)$ for almost everywhere $t \in\left[t_{0}, T\right]$ and $\bar{x}(t)$ can be described as follows:

(a) If $T-t_{0} \leq a^{-1}\left(1+x_{0}\right)$, then $\bar{x}(t)$ is given by (3.53).

(b) If $a^{-1}\left(1+x_{0}\right)<T-t_{0}<2 \rho-a^{-1}\left(1+x_{0}\right)$, then $\bar{x}(t)$ is given by (3.55).

(c) If $2 \rho-a^{-1}\left(1+x_{0}\right)<T-t_{0}<\rho+a^{-1}\left(1-x_{0}\right)$, then $\bar{x}(t)$ is given by either (3.54), or (3.55).

(d) If $\rho+a^{-1}\left(1-x_{0}\right) \leq T-t_{0}<a^{-1}\left(3-x_{0}\right)$, then $\bar{x}(t)$ is given by either (3.55), or (3.56).

(e) If $T-t_{0}=a^{-1}\left(3-x_{0}\right)$, then $\bar{x}(t)$ is given by (3.57).

(f) If $T-t_{0}>a^{-1}\left(3-x_{0}\right)$, then $\bar{x}(t)$ is given by (3.58) .

In the situations described in (a), (b), (e), and $(\mathrm{f}),(\bar{x}, \bar{u})$ is a unique local solution of $\left(F P_{3}\right)$, which is also a unique global solution of the problem.

Proof. Suppose that $\rho<2 a^{-1}, x_{0}>-1$, and $a^{-1}\left(1+x_{0}\right) \leq \rho$. Let $\rho_{1}, \rho_{2}$ be given by (3.43). Then, combining the results formulated in Case 1 and Case 3, and noting that the function $\bar{x}_{1}(t)$ in $\left(F P_{3 a}\right)$ plays the role of $\bar{x}(t)$ in $\left(F P_{3}\right)$, we obtain the assertions $(\mathrm{a})-(\mathrm{f})$.

\section{Conclusions}

We have analyzed a maximum principle for finite horizon state constrained problems via one parametric example of optimal control problems of the Langrange type, which has five parameters. This problem resembles the optimal growth problem in mathematical economics. It belongs to the class of control problems with bilateral state constraints. We have proved that the control problem in the example can have not more than two local solutions, and at least one of them which must be a global solution. Moreover, we have presented explicit descriptions of the optimal processes, which are suspected to be local solutions, with respect to the five parameters. 
The obtained results allow us to have a deep understanding of the maximum principle in question.

It seems to us that economic optimal growth models can be studied by advanced tools from functional analysis and optimal control theory via the approach adopted in this paper.

\section{References}

[1] L. S. Pontryagin, V. G. Boltyanskii, R. V. Gamkrelidze, E. F. Mishchenko, The Mathematical Theory of Optimal Processes, Interscience Publishers John Wiley \& Sons, Inc., New York-London, 1962.

[2] R. F. Hartl, S. P. Sethi, R. G. Vickson, A survey of the maximum principles for optimal control problems with state constraints, SIAM Rev. 37 (1995), 181-218.

[3] A. D. Ioffe, V. M. Tihomirov, Theory of Extremal Problems, North-Holland Publishing Co., Amsterdam-New York, 1979.

[4] H. X. Phu, A solution method for regular optimal control problems with state constraints, J. Optim. Theory Appl. 62 (1989), 489-513.

[5] H. X. Phu, Investigation of a macroeconomic model by the method of region analysis, J. Optim. Theory Appl. 72 (1992), 319-332.

[6] R. Vinter, Optimal Control, Birkhäuser, Boston, 2000.

[7] V. Basco, P. Cannarsa, H. Frankowska, Necessary conditions for infinite horizon optimal control problems with state constraints, Math. Control Relat. Fields 8, 535-555 (2018).

[8] A. Takayama, Mathematical Economics, The Dryden Press, Hinsdale, Illinois, 1974.

[9] L. Cesari, Optimization Theory and Applications, 1st edition, Springer-Verlag, New York, 1983.

[10] B. S. Mordukhovich, Variational Analysis and Generalized Differentiation, Vol. I: Basic Theory, Springer, New York, 2006.

[11] B. S. Mordukhovich, Variational Analysis and Generalized Differentiation, Vol. II: Applications, Springer, New York, 2006.

[12] B.S. Mordukhovich, Variational Analysis and Applications, Springer, Berlin, Switzerland, 2018. 
[13] V. T. Huong, J.-C. Yao, N. D. Yen, Analyzing a maximum principle for finite horizon state constrained problems via parametric examples. Part 1: Problems with unilateral state constraints, arXiv:1901.03794 (2019).

[14] A. N. Kolmogorov, S. V. Fomin, Introductory Real Analysis. Revised English edition. Translated from the Russian and edited by R. A. Silverman, Dovers Publications, Inc., New York, 1970.

[15] D. G. Luenberger, Optimization by Vector Space Methods. John Wiley \& Sons, New York, 1969.

[16] H. L. Royden, P. M. Fitzpatrick, Real Analysis. Fourth edition. China Machine Press, 2010 . 